\title{
Faire oeuvre de pionnier: Atteindre les femmes agriculteurs en
} Zambie occidentale

Janice Jiggins

Paul Maimbo

Mary Masona

Follow this and additional works at: https://knowledgecommons.popcouncil.org/departments_sbsr-pgy

Part of the Agricultural and Resource Economics Commons, Family, Life Course, and Society

Commons, Gender and Sexuality Commons, Inequality and Stratification Commons, and the International

Public Health Commons

How does access to this work benefit you? Let us know!

\section{Recommended Citation}

Jiggins, Janice, Paul Maimbo, and Mary Masona. 1992. "Faire oeuvre de pionnier: Atteindre les femmes agriculteurs en Zambie occidentale," SEEDS no. 14. New York: Population Council. 


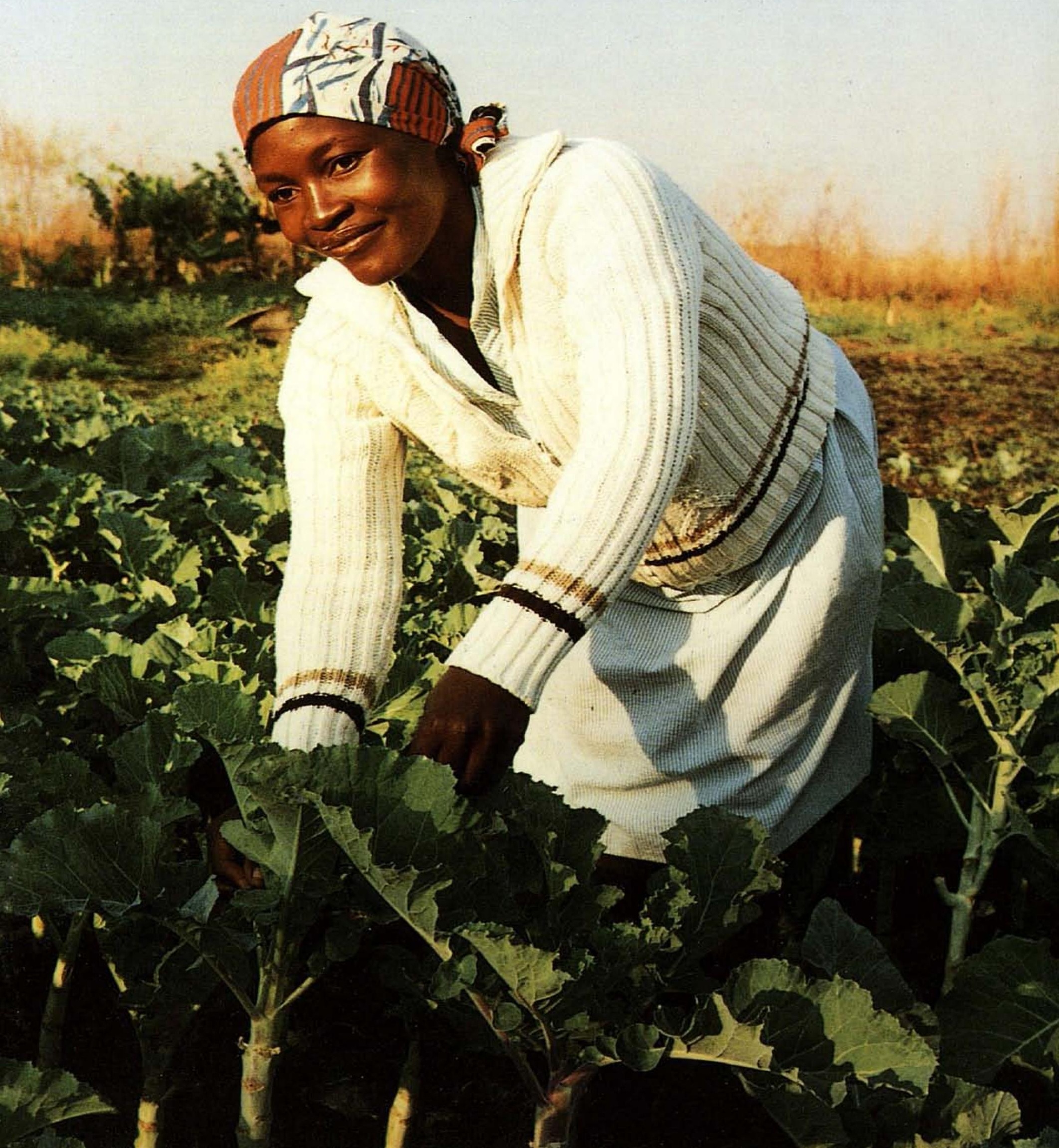


SEEDS est une série de brochures publiée en réponse aux demandes d'information venant du monde entier sur des idées de programmes innovatrices et pratiques, développées pour aborder le problème de la condition et des besoins économiques des femmes aux revenus faibles. Le but de ces brochures est de diffuser l'information et de stimuler la création de nouvelles initiatives fondées sur les expériences positives de projets visant à aider les femmes à gagner leur vie et améliorer leur statut économique. Les projets décrits dans ce numéro comme dans le reste de la série ont été choisis du fait qu'ils servent non seulement à renforcer les rôles producteurs des femmes mais aussi à assurer leur intégration dans différents secteurs de développement à la fois sur le plan économique et social. Tous les projets publiés dans le cadre de la série SEEDS associent les femmes aux prises de décision, organisent les femmes au niveau local et abordent les problèmes de politique générale qui influent sur les rôles économiques des femmes.

Ces rapports n'ont cependant pas été conçus pour être suivis à la lettre car chaque effort de développement doit faire face à des problèmes et des possibilités quelque peu différents. En revanche, ils relatent l'histoire d'une idée et sa mise en application avec l'espoir que les leçons apprises se révéleront utiles dans des environnements variés. C'est également pour être portés à l'attention de ceux qui détiennent les pouvoirs de décision qu'ils ont été rédigés et leur montrer que les femmes jouent des rôles vitaux non seulement dans l'économie de leurs ménages mais également dans la vie économique de chaque nation.

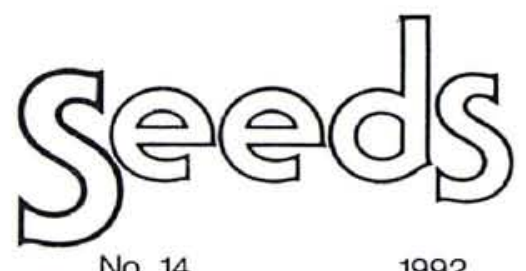

ISSN $073-6833$

Copyright (C) 1992 SEEDS
La publication de SEEDS est administrée par le Population Council. Les critères de publication sont déterminés par le Comité de Rédaction de SEEDS: Judith Bruce (Population Council). Betsy Campbell (The Ford Foundation). Marty Chen (Harvard Institute for International Development), Anne Kubisch (The Ford Foundation).

Ann Leonard (The Population Councilj). Cecilia Lotse (UNICEF), Katharine McKee (Center for Community Self-Help), Anne Walker (International Women's Tribune Center) et Mildred Warner (Cornell University).

La traduction française de ce numéro de SEEDS a été subventionnée par la Foundation Rockefeller et le Population Council.

Les opinions et commentaires exprimés dans cette brochure sont la seule responsabilité des auteurs et n'engagent qu'eux. 


\title{
Faire Oeuvre de Pionnier: Atteindre les Femmes Agriculteurs en Zambie Occidentale
}

\author{
par Janice Jiggins \\ avec Paul Maimbo et Mary Masona
}

\section{Introduction}

En Afrique Sub-saharienne, plus de 60\% de la nourriture destinée à la consommation et à la vente de même qu'une large proportion des cultures commerciales non alimentaires sont produites par les femmes. Toutefois, en dépit du rôle crucial que jouent les femmes dans la production agricole, la transformation et la vente des produits agricoles en Afrique (tout comme dans d'autres régions du monde), celles-ci ne bénéficient presque d'aucun soutien de la part des pouvoirs publics. Au cours de ces dernières décennies, des projets à petite-échelle furent mis en place sur l'ensemble du continent africain pour tenter d'incorporer les femmes aux politiques de développement agricole. Pourtant, à l'heure qu'il est, seul une poignée de ministères de l'agriculture ont véritablement tiré les leçons de ces expériences. Dans ce numéro de SEEDS, nous vous présentons un exemple particulièrement éloquent qui nous vient de la Province Occidentale de la Zambie. Le projet en question vise à opérer un changement radical de la manière dont fonctionne une bureaucratie agricole en redéfinissant les mandats, en encourageant un changement d'attitude par le biais de programmes de formation du personnel, en favorisant les possibilités de développement au niveau des villages tout en étant plus sensible aux besoins des villageois et, finalement, en multipliant les programmes de recherche économique et technique. Leur expérience démontre que pour réussir à atteindre les femmes et répondre à leurs besoins, une variété d'approches sont nécessaires. 


\section{Contexte}

La Zambie est un pays pauvre, enclavé, qui se situe au centre-sud de l'Afrique. Pour les habitants de Lusaka, la capitale, la Province Occidentale est un endroit isolé, dépourvu des rudiments de la vie moderne. Mongu, la capitale de la Province, se situe à plus de $600 \mathrm{~km}$ de Lusaka et est accessible par bus ou par voiture durant la saison sèche. Mais avec la venue des pluies le trajet devient de plus en plus difficile et les vols hebdomaires sont bien trop chers pour la majorité des Zambiens.

En voyageant d'Est en Ouest, le paysage se transforme lentement d'un plateau sablonneux légèrement boisé en escarpement à Mongu, où la terre chute abruptement pour faire place à la plaine inondable du Zambèze. Au cours de la saison des pluies, on ne peut traverser la plaine qu'en bateau ou en canoe. La fertilité de la terre est variée et s'étend de la région relativement prospère de Kaoma sur le plateau, un centre de production de maïs, au district frappé par la famine de Senanga Ouest dans l'extrême sud du pays.

Au cours de ces dernières années, les petits cultivateurs de la Province Occidentale ont subi les contrecoups de la mauvaise situation économique du pays. Presque rien ne fonctionne et la plupart des choses, depuis les pompes à eau jusqu'aux cliniques, sont sous-utilisées en raison de l'effondrement des services publics et du man- que de pièces de rechange, d'entretien et d'approvisionnement. Les subventions alimentaires ont également connu un fort déclin et les intrants comme l'engrais, par exemple, sont de plus en plus difficiles à se procurer. De nombreux employés touchent des allocations sociales ou des rations alimentaires en plus de leurs salaires mais la création d'emplois est limitée tant dans le secteur public que privé. Cette situation est particulièrement éprouvante pour les femmes-quelles que soient leurs catégories sociales-puisqu'elles doivent soigner les malades, nourrir les chômeurs et essayer d'amasser suffisamment d'argent pour envoyer leurs enfants à l'école en dépit de la crise économique dans lequel est plongé le pays.

\section{Les Femmes de la Province Occidentale}

Comme presque toutes les femmes en Zambie rurale, la majorité des femmes de la Province Occidentale travaillent la terre:

- Par tradition. II est du devoir d'une femme de cultiver la terre pour se nourrir elle-même et ses enfants et pour vendre les produits de la ferme pour acheter des produits de première nécessité. Elle doit également servir comme principale source de main d'oeuvre pour soutenir les activités agricoles de son mari et de sa famille;

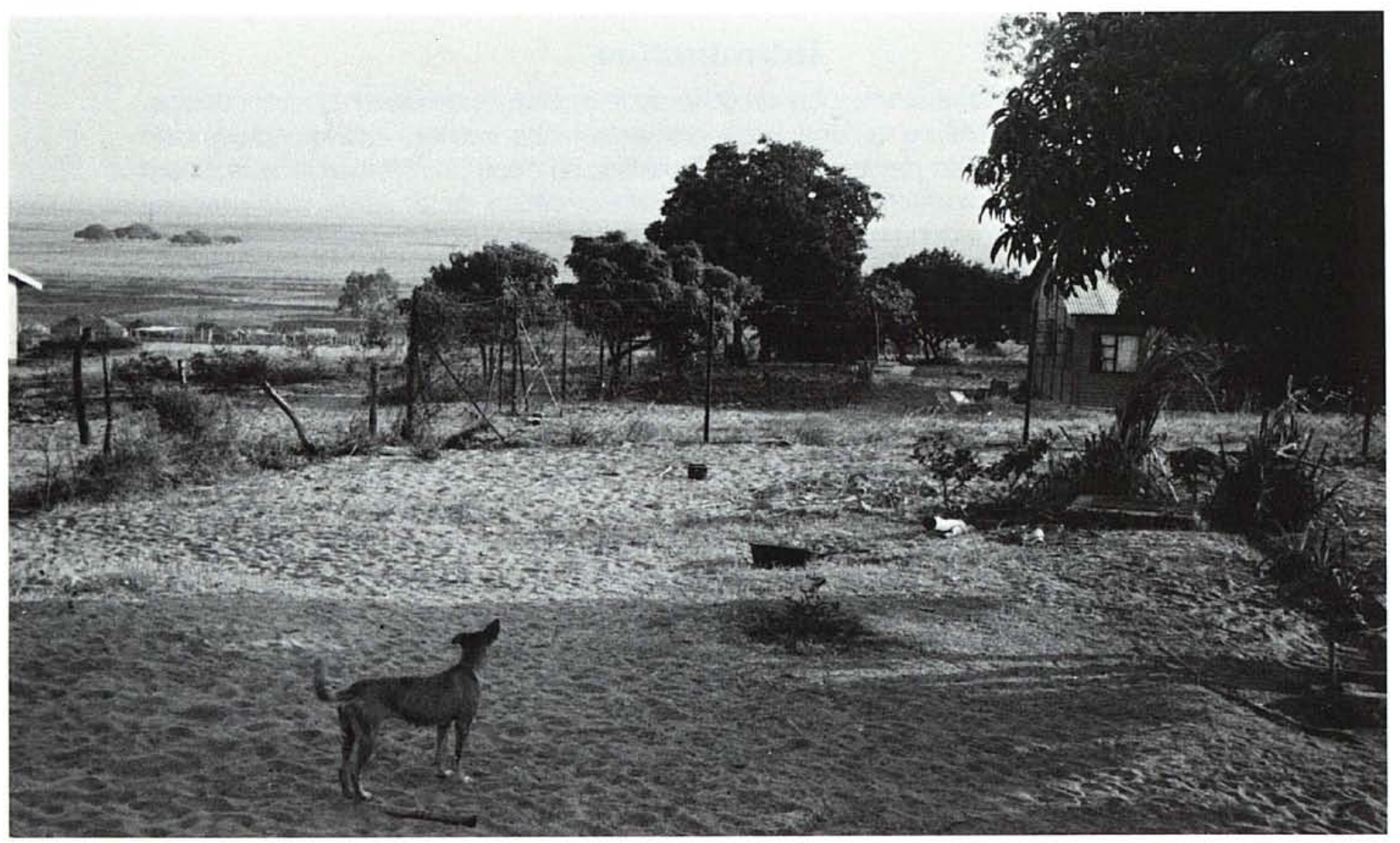


- Par nécessité. En Zambie, plus d'une femme sur trois, de droit ou de fait, est seule pour assurer la subsistance de sa famille. Certaines données obtenues lors du dernier recensement semblent indiquer que le nombre de femmes chefs de cellule agricole familiale est plus important dans les régions rurales les plus pauvres et les plus isolées où l'agriculture est l'unique moyen de subsistance; dans certains districts, plus de $60 \%$ des ménages ont comme seul soutien économique une femme.

- Par choix. Dans les endroits qui possèdent de la bonne terre des services de conseils en agriculture efficaces, des marchés facilement accessibles, des approvisionnements d'engrais réguliers et un réseau routier ou ferroviaire adéquat, l'introduction de cultures destinées exclusivement à la vente, comme, par exemple, l'arachide, peuvent constituer pour les femmes et les hommes des sources de revenus appréciables.

En Zambie occidentale, tout comme dans nombre de sociétés africaines, la condition de la femme et son accès à la terre sont fonction de son marriage, de sa famille et de sa capacité à avoir des enfants. Dans la Province Occidentale, entre un cinquième et un quart de toutes les femmes mariées vivent dans des unions polygames. Les co-épouses ne résident habituellement pas dans le même village et reçoivent la visite de leur mari à tour de rôle. Les villages ont tendance à être petits, ne comprenant en moyenne pas plus d'une centaine d'habitants, et sont très dispersés. Avec peut-être moins de vingt ménages par village et la plupart des hommes absents à la recherche d'un emploi rémunéré, la majorité des femmes adultes sont seules pour assurer la subsistance de leur famille et une large proportion d'entre elles sont des divorcées.

Une des conséquences de cette situation est que le soutien traditionnellement apporté par la collectivité aux familles les plus vulnérables devient de plus en plus faible et que les femmes élevant seules leurs enfants sont particulièrement vulnérables; elles deviennent plus isolées socialement et n'ont presque pas de membres de la famille vers qui se tourner en cas de besoin immédiat comme, par exemple, pour avancer de l'argent, fournir de la main d'oeuvre ou aider avec les corvées domestiques. Dans de telles circonstances, les femmes souvent éprouvent un sentiment d'abandon et de désespoir. C'est ainsi qu'une femme de Kweseka, un village situé dans

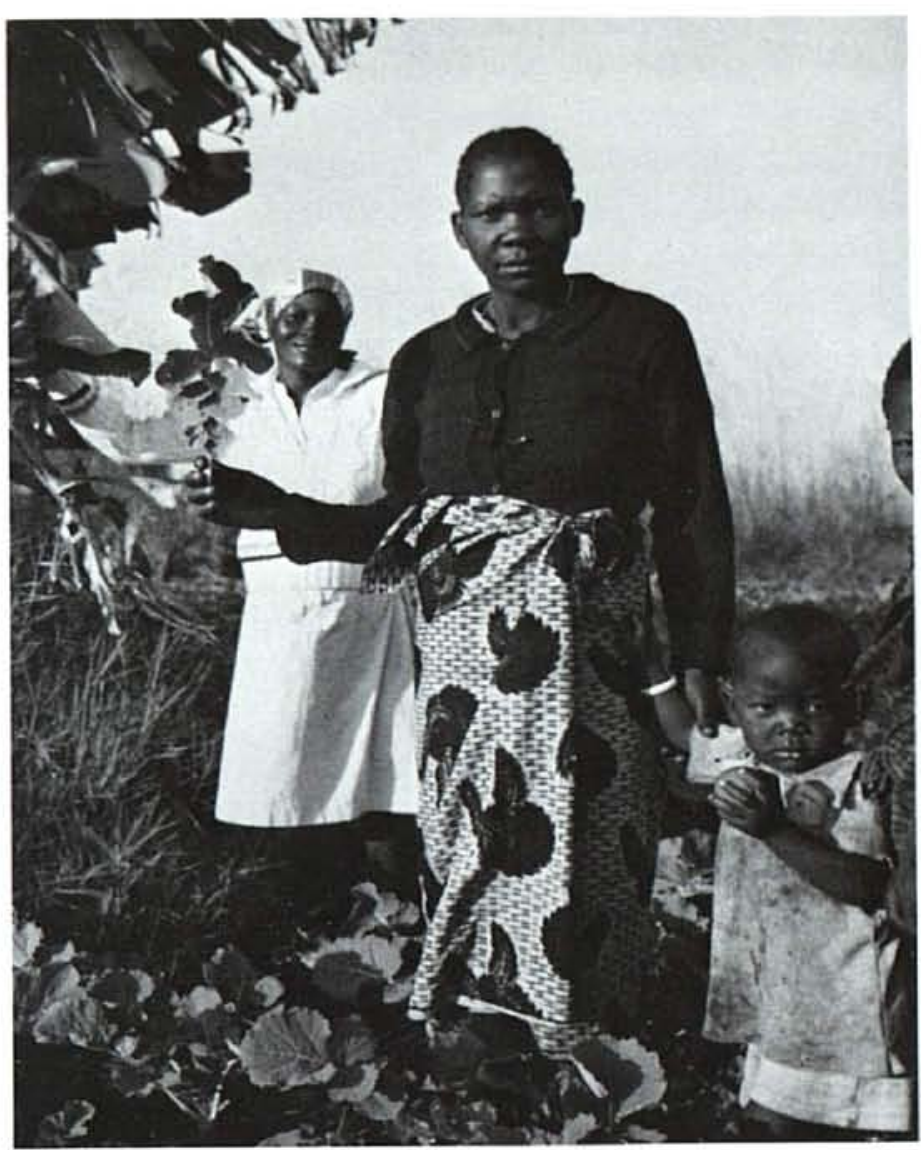

le district de Mongu, se lamente: "Le monde nous a oublié".

Dans la Province Occidentale, les dirigeants des collectivités locales déterminent le plan d'occupation du sol. Etant donné que la province est peu peuplée, les hommes et les femmes peuvent facilement obtenir des lopins de terre pour la culture et le patûrage mais il existe tout de même une concurrence localisée pour les champs qui se trouvent dans les régions les plus fertiles et les mieux accessibles. Dans cette partie de la Zambie, le principal obstacle à la production agricole n'est pas le manque de terre arable mais le manque de main d'oeuvre-plus particulièrement le manque de main d'oeuvre féminine. Les femmes constituent la principale source de main d'oeuvre agricole dans la Province Occidentale, et les hommes de même que les autres membres de la famille ont habituellement la priorité sur l'utilisation de cette main d'oeuvre. II est vrai que certains hommes aident les femmes à défricher des champs pour planter des cultures vivrières mais ces derniers sont rares. En fait, la majorité des hommes passent presque tout leur temps à s'occuper de leur bétail, à pêcher ou encore à planter leurs propres cultures vivrières ou, dans certains cas, des cultures commerciales.

Plusieurs études ont révélé que, durant la courte saison des pluies, les femmes travaillent 
plus de dix heures par jour pour attenir aux corvées agricoles et domestiques tandis que les hommes passent considérablement moins de temps à travailler dans les champs. En raison de cette division du travail traditionnelle, il peut arriver qu'une femme mariée se trouve à court de main d'oeuvre même lorsque le mari demeure avec elle. C'est une des raisons pour lesquelles, les femmes souvent forment des associations pour échanger leur capacité de travail à des fins d'assistance mutuelle puisque très peu d'entre elles gagnent suffisamment d'argent pour embaucher de la main d'oeuvre. En fait, la plupart des femmes qui louent leur force de travail sont des femmes chefs de famille; mais, le peu d'argent qu'elles gagnent sert à acheter de la nourriture pour compenser les maigres récoltes qu'elles tirent de leurs propres cultures étant donné qu'elles n'ont pas le temps de s'en occuper correctement.

La préparation des aliments est également une tâche très prenante pour les femmes. La denrée de base-qu'il s'agisse du maïs, du millet, du sorgho ou du manioc-est d'abord réduite en farine puis consommée sous forme de bouillie épaisse. Etant donné que les broyeurs à marteaux sont rares et que le pilage à la main est un travail

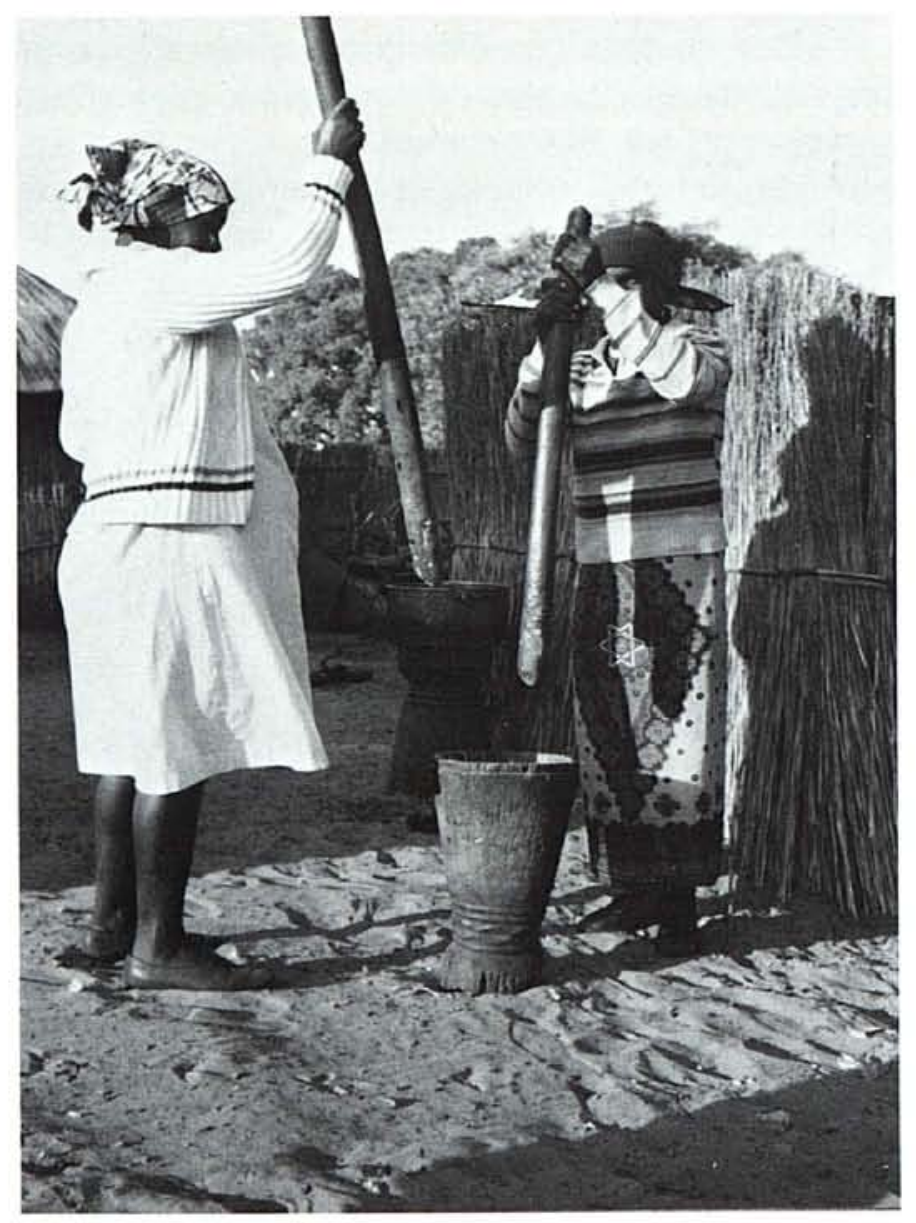

fastidieux, les ménages préfèrent, quand c'est possible, vendre leurs récoltes et acheter à la place des céréales raffinées, en l'occurence de la farine de maïs moulue. En raison du temps nécessaire à sa tranformation, le riz est rarement consommé à la maison; la récolte est généralement vendue dans son intégralité. Les femmes doivent aussi consacrer du temps à d'autres tâches domestiques, comme, par exemple, chercher de l'eau et du bois de feu et s'occuper des enfants.

En Zambie occidentale, il existe très peu de sources de revenus pour les femmes en dehors de l'agriculture. Certaines d'entre elles gagnent un peu d'argent de temps à autre en vendant des nattes et des paniers, des fruits sauvages et des champignons, des bananes, des mangues ou du poisson. Plusieurs études indiquent que les femmes utilisent leur revenu pour acheter, par ordre d'importance, de la nourriture, des vêtements et pour payer les frais de scolarité de leurs enfants.

La fabrication et la vente de bière est de loin la source de revenu la plus répandue parmi les femmes. Selon plusieurs enquêtes, plus de troisquarts des femmes font régulièrement de la bière, principalement, pour la vendre mais aussi pour mobiliser et récompenser les travailleurs journaliers. En moyenne, les femmes brassent de la bière quatre à cinq fois par an, généralement durant la saison sèche. Les bénéfices nets sont très variables mais il arrive fréquemment qu'un brassin rapporte jusqu'à 100 kwacha (2-3 dollars US).

En Zambie occidentale, des contraintes au niveau du temps que les femmes peuvent consacrer à la production de cultures vivrières ainsi qu'un manque d'argent les empêchent de se procurer les aliments nécessaires à assurer une bonne nutrition. En fait, les femmes cultivent presque exclusivement du manioc qui leur sert de nourriture et leur permet également d'en tirer un revenu puisqu'elles en font de la bière. Le manioc est une plante relativement résistante qui ne demande aucun travail particulier pour pousser et reste en terre aussi longtemps que nécessaire. Bien qu'il soit riche en féculent, c'est un aliment pauvre en protéines et en minéraux. Donc, en plus de la sous-nutrition déjà très répandue dans la région, on observe de plus de plus de cas de malnutrition, plus particulièrement, parmi les enfants qui n'aiment pas la consistance épaisse ni le goût farineux du manioc et n'en consomment pas suffisamment pour assurer l'équilibre nutritionnel. 


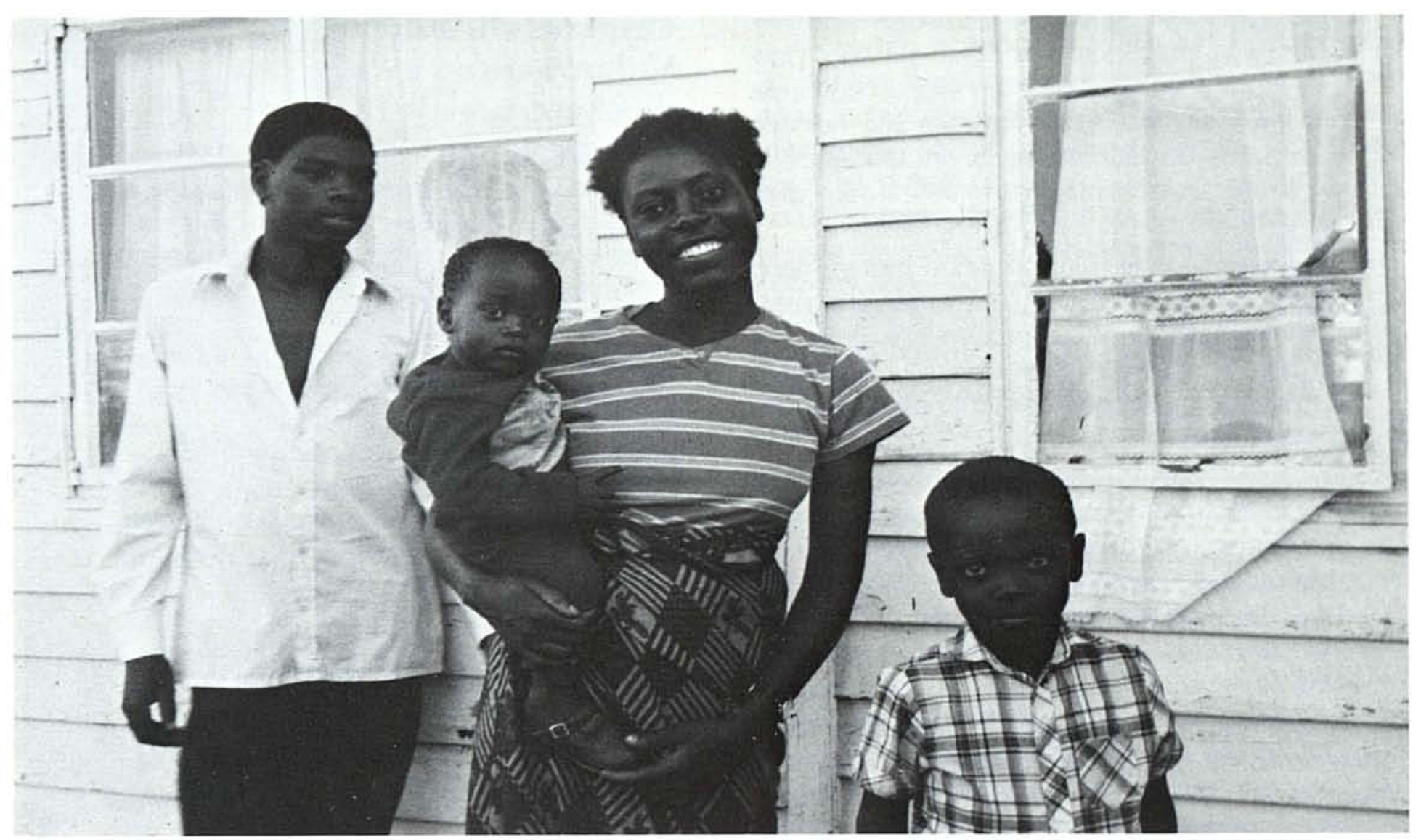

\section{Vulgarisation Agricole et Femmes Agriculteurs}

En Zambie, tout comme dans le reste de l'Afrique, les pouvoirs publics ont négligé le rôle important que jouent les femmes dans l'agriculture parce que la plupart des décideurs, des gestionnaires et des planificateurs sont des hommes qui considèrent que le travail des femmes dans les champs est tout simplement "le lot des femmes". La contribution réelle de l'agriculture féminine au maintien du bien-être familial et de la sécurité alimentaire du pays est restée virtuellement invisible. Par ailleurs, en raison des idées pré-conçues du gouvernement et des agences d'assistance technique pour qui agriculture moderne équivaut à agriculture "masculine", les enquêtes sur l'agriculture et l'élevage ne recueillent que très rarement des données sur des questions ayant trait à la part de travail des femmes, aux techniques de gestion des femmes ou encore aux cultures produites uniquement par les femmes.

Bien que les femmes continuent à ne pas être associées aux initiatives de développement agricole, celles-ci jouent pourtant un rôle de plus en plus crucial. Des changements complexes dans la structure économique et sociale font que de plus en plus de femmes rurales se retrouvent désormais seules pour assurer la subsistance de leur famille. Quoique toutes les femmes chefs de cellule agricole familiale ne partagent pas les mêmes problèmes, les contraintes en temps et en énergie que cette situation engendre sont telles que la plupart des femmes finissent par se retrouver isolées du point de vue social et marginalisées du point de vue économique. Le travail dans les champs, la charge des enfants et d'autres membres de la famille exigent des femmes tellement d'énergie qu'elles ont du mal à fonctionner ce qui a pour conséquence la déterioration rapide du bien-être et de la sécurité de la famille.

Quoique nombre de femmes rurales arrivent à s'en sortir, la majorité d'entre elles sont confrontées à de nombreuses difficultés auxquelles les pouvoirs publics sont lents à répondre faute de les déceler à temps. Parmi ces difficultés, les plus importantes sont les suivantes:

- Journées de travail très longues et trop chargées et peu d'accès à l'argent nécessaire pour embaucher de la main d'oeuvre;

- Manque d'accès au crédit et aux services financiers qui est généralement lié à des problèmes de transports, au statut juridique de la femme et aux types de services financiers offerts; 
- Le sexe des agents de formation agricole (principalement des hommes) qui sont peu disposés à transgresser les tabous culturels en travaillant avec des femmes qui ne font pas partie de leur famille et ont tendance à avoir des opinions bien arrêtées sur ce que les femmes peuvent et doivent faire.

- Manque d'accès à la formation (notamment dans les domaines d'activités critiques comme l'usage de la traction animale) aux engrais et autres matériels ce qui est aussi imputable au sexe des agents de vulgarisation agricole; et

- Les femmes elles-mêmes qui très souvent ne possèdent pas l'assurance nécessaire pour s'affirmer et revendiquer leurs droits, assumer de nouvelles responsabilités ou faire évoluer les mentalités en dépit des changements importants de la structure sociale.

Bien que le gouvernement Zambien soutienne dans ses grandes lignes les efforts visant à améliorer la condition de la femme, la politique agricole ne reconnait toujours pas explicitement le rôle important que jouent les femmes dans l'agriculture. Depuis le milieu des années 80 , des chercheurs femmes de l'Université de Zambie, des responsables du projet "Femmes et Développement" et le chef du service "Economie ménagère" du Ministère de l'Agriculture et de l'Aménagement des Eaux travaillent en collaboration pour développer des idées et des stratégies visant à: 1) identifier les changements politiques souhaitables; 2) attirer l'attention des pouvoirs publics sur des projets pertinants; et 3) faire en sorte que la contribution des femmes à l'agriculture soit officiellement reconnue et que ces dernières soient étroitement associées aux projets de développement agricole du gouvernement. Ce groupe de travail suit de très près les activités du Programme de Vulgarisation à l'intention des Femmes (WEP) en Zambie Occidentale puisque, contrairement à la plupart des autres projets de vulgarisation à l'intention des femmes, son objectif principal n'est pas de fournir des services directement aux femmes travaillant la terre. II s'agit plutôt d'une intervention stratégique de durée limitée dont le but est de modifier la manière dont opèrent les programmes du département de l'agriculture, notamment, à travers des recommandations, des programmes de formation, des campagnes d'information, des données statistiques, la persuasion et une collaboration étroite entre les différents participants.

\section{Origines du Programme de Vulgarisation à l'Intention des Femmes (WEP)}

Bien que le Programme de Vulgarisation à l'intention des Femmes (WEP) opère à partir des locaux de la division "Economie ménagère" du Département de l'Agriculture, c'est le responsable agricole provincial qui assure la direction générale de ses activités. L'autorité du responsable agricole provincial permet au WEP, à son tour, d'avoir accès aux activités de formation et de vulgarisation de l'ensemble des divisions du Département ainsi qu'aux activités de la division Recherche. La localisation du programme au sein de la division "Economie ménagère" et sa conception ne sont pas le fait du hasard. Plusieurs expérimentations ont révélées qu'ainsi conçu, le programme avait les meilleures chances de développer des liens étroits avec l'ensemble des divisions du département et de mettre en place des méthodes permettant effectivement de changer les mentalités.

Depuis 1971, la division "Economie ménagère" organise des cours de formation à l'intention des femmes sur des sujets relatifs à la conservation et l'entreposage des aliments, la nutrition et les soins à donner aux enfants mais en ce qui concerne les activités agricoles des femmes, la division s'est heurtée à deux obstacles: d'une part, la nécessité de faire appel à des spécialistes d'autres divisions pour les questions d'assistance technique et, d'autre part, le besoin d'utiliser les agents de terrain du département pour atteindre les femmes. Donc pour arriver à réaliser ses objectifs, il a fallu que la division développe des méchanismes lui permettant de travailler efficacement avec des tierces personnes dont la plupart étaient des hommes.

Dans la Province Occidentale, la plupart des 200 agents de terrain, appelés "assistants agricoles" sont des hommes. Le responsable provincial de la section "Economie ménagère", Mme Elizabeth Kazungo, remarque: "En fait, cela a toujours été très difficile. Les hommes estiment que tout ce qui touchent à l'agriculture féminine et à l'économie ménagère n'est pas de leur ressort."

En outre, dans un système reposant sur des visites indivuelles à chaque ferme, le nombre d'assistants agricoles est bien trop faible pour assurer une couverture adéquate. Le ratio d'assistants par ménages se situe aux alentours de 1 pour 450 . Une enquête réalisée dans cinq régions de la Province Occidentale a révélée que tandis que 40 pourcent des foyers dirigés par un homme ne bénéficient d'aucune visite, plus de 80 pourcent 


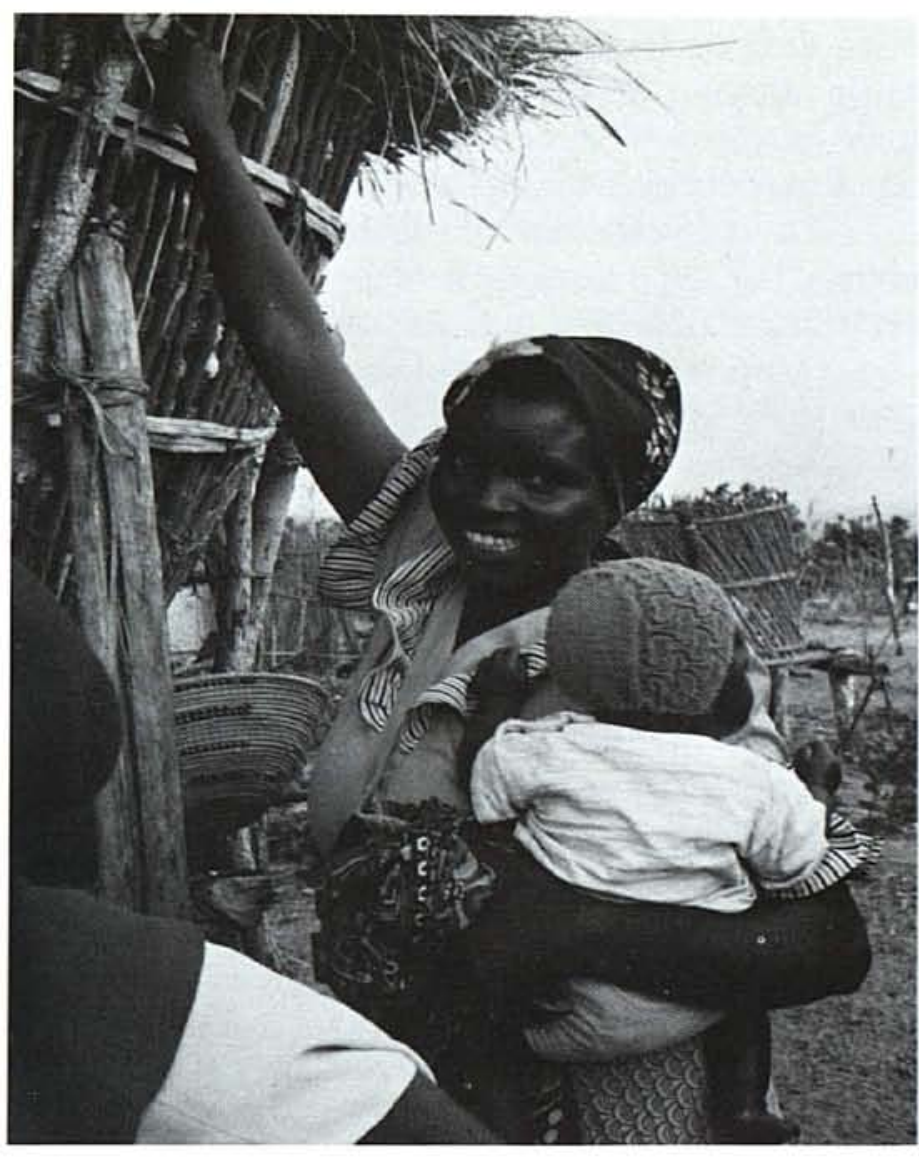

des ménages dirigés par une femme ne sont jamais visités. Les assistants agricoles ont tendance à excuser cette négligence des femmes agriculteurs en se cachant derrière la tradition. Ils affirment: "Le fait de parler à une femme nous met mal à l'aise. Ce n'est pas notre coutume. Les maris et les petits amis pourraient nous accuser."

Une véritable percée s'est produite au début des années 80 lorsque le Département, avec le soutien du gouvernement néerlandais, démarra un projet spécial pour cultiver du riz. Les responsables du projet se rendirent très vite compte que, pour réussir, il fallait associer les femmes plus étroitement aux activités prévues. De ce fait, on embaucha une spécialiste hollandaise pour mener une enquête auprès des femmes locales afin d'obtenir des informations sur leur charge de travail, leur contribution possible à la production de riz et leurs sentiments à l'égard de l'éducation en matière agricole et de l'accès aux assistants agricoles de sexe masculin. Son rapport contenait les données de base nécessaires pour entamer des discussions sur les femmes agriculteurs et leurs besoins. Par ailleurs, la présence d'un "expert" de sexe féminin fit prendre conscience aux membres du personnel autochtones et internationaux que les questions touchant à la participation des femmes à l'agriculture étaient à prendre au sérieux.
Une fois l'enquête terminée, on demanda à la spécialiste hollandaise d'organiser les femmes qui allaient participer au projet en petits groupes dans le but de faciliter leur formation. Elle commença par recruter plusieurs femmes travaillant pour le projet comme agent de vulgarisation comme Mme Masona qui plus tard fut nommée à la tête du WEP. Mme Masona travaillait alors comme conseillère en élevage et avait pour mission d'évaluer le nombre de boeufs nécessaires pour labourer les rizières. Ensemble, elles commencèrent par organiser les femmes agriculteurs en petits groupes. L'idée prévalait également que les assistants agricoles seraient moins réticents à travailler avec des groupes de femmes qu'avec des individus.

L'approche de groupe a été une réussite totale. Comme le dit Mme Kayombo: "Ceci correspond tout à fait à notre façon de faire les choses. Dans très peu de temps, nous nous sentirons entièrement à l'aise. II y a tellement de solidarité entre les gens." Mais cette expérience a également convaincu le Département qu'une intervention plus stratégique était nécessaire pour surmonter certains obstacles relatifs à l'attitude des membres du personnel et un certain nombre de difficultés d'ordre pratique. Après plus amples réflexion et analyse, il a été décidé que le programme de formation pour les femmes-bien que situé au sein de la division "Economie ménagère"-allait être rattaché au programme de vulgarisation en cours puisque cela permettrait au WEP de renforcer ses liens avec l'ensemble des programmes de vulgarisation agricole du Département.

Ce nouveau programme avait deux objectifs principaux concernant la formation. Le premier était d'ordre interne et consistait à ajouter au programme de formation des assistants agricoles un cours pour les sensibiliser sur le rôle des femmes de la Province Occidentale dans l'agriculture. Le second, d'ordre externe, était de développer et de diriger des cours de formation "à domicile" pour les femmes portant exclusivement sur l'agriculture féminine. En 1986, Mme Mary Masona devint la première coordinatrice du WEP.

C'était un véritable défi mais on n'a pas le temps de s'ennuyer - il y a tellement à faire! De plus, je viens de cette région et je connais les traditions. J'ai dix enfants et je connais les difficultés auxquelles sont confrontées les femmes d'ici."

Mary Masona 
Le WEP s'est fixé les objectifs suivants:

- recueillir des informations sur les activités agricoles des femmes et les contraintes auxquelles elles sont soumises;

- faire comprendre aux membres du département l'importance d'atteindre les femmes agriculteurs;

- formuler des recommendations sur les meilleures méthodes pour atteindre les femmes rurales;

- sensibiliser les agents de terrain sur l'importance des activités agricoles des femmes;

- contribuer à la conception et la mise en place de projets de développement et procéder à l'évaluation de leurs effets sur les femmes;

- participer à l'organisation et à la gestion des groupes de femmes agriculteurs

\section{Faire Figurer les Femmes à l'Ordre du Jour}

Le WEP exerce ses activités principalement à travers les structures, le personnel et les projets existants du Département de l'Agriculture. Mise à part le coordinateur, le WEP n'a pas d'employés à proprement parler. Son influence est plus basé sur la concertation et la coopération que sur l'autorité formelle et le contexte bureaucratique déter- mine la manière dont fonctionne le programme. Mme Masona définit le WEP comme un "catalyseur stratégique" pour aider les femmes à accéder à la reconnaissance sociale et économique.

Le coordinateur travaille donc par l'entremise de ses collègues provinciaux et de district pour créer des liens avec les autres divisions en vue d'obtenir leur compréhension et leur soutien. Une première étape importante a été de faire prendre conscience aux collègues travaillant dans les districts et sur le terrain de l'importance des activités agricoles des femmes dans la province.

Comité de Coordination Départemental.

Le Comité de Coordination Départemental (composé de chefs de division et de coordinateurs de projets) est un forum important pour maintenir l'intérêt dans les objectifs du WEP. Le comité essaie de se rencontrer sur une base régulière pour faire le point de la situation, échanger des idées et revoir leurs stratégies. Initialement, on comptait sur les femmes du comité pour trouver des idées sur la façon d'atteindre les femmes rurales. Mais, comme le fait remarquer Mme Kayombo, les collègues de sexe masculin ont pris suffisamment d'assurance pour apporter leurs propres suggestions. En évitant les confrontations et en se concentrant sur la présentation de données et de résultats d'études sur le terrain, le WEP a également été en mesure de modifier quelque peu les rapports hommes-femmes au cours des réunions

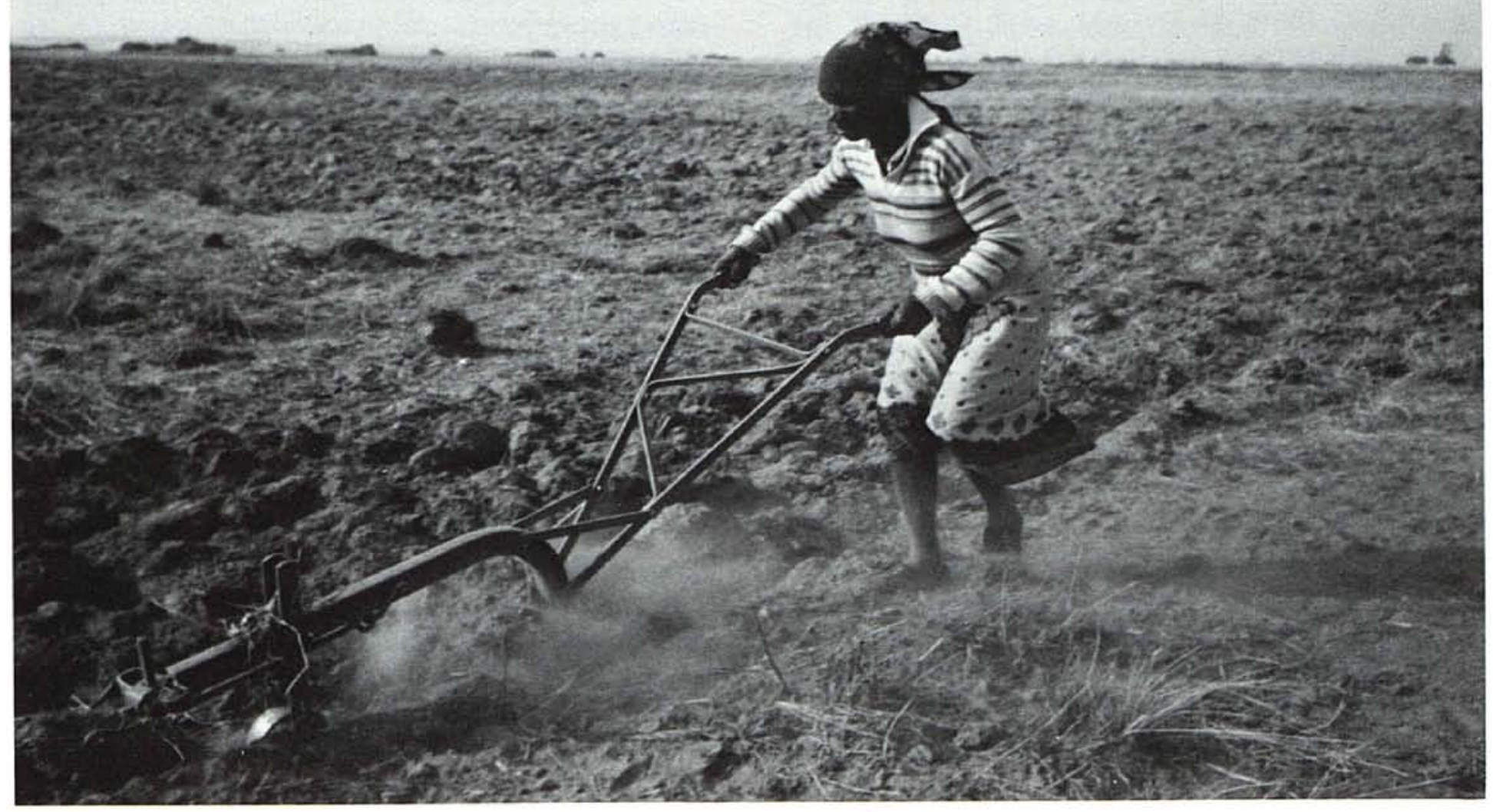




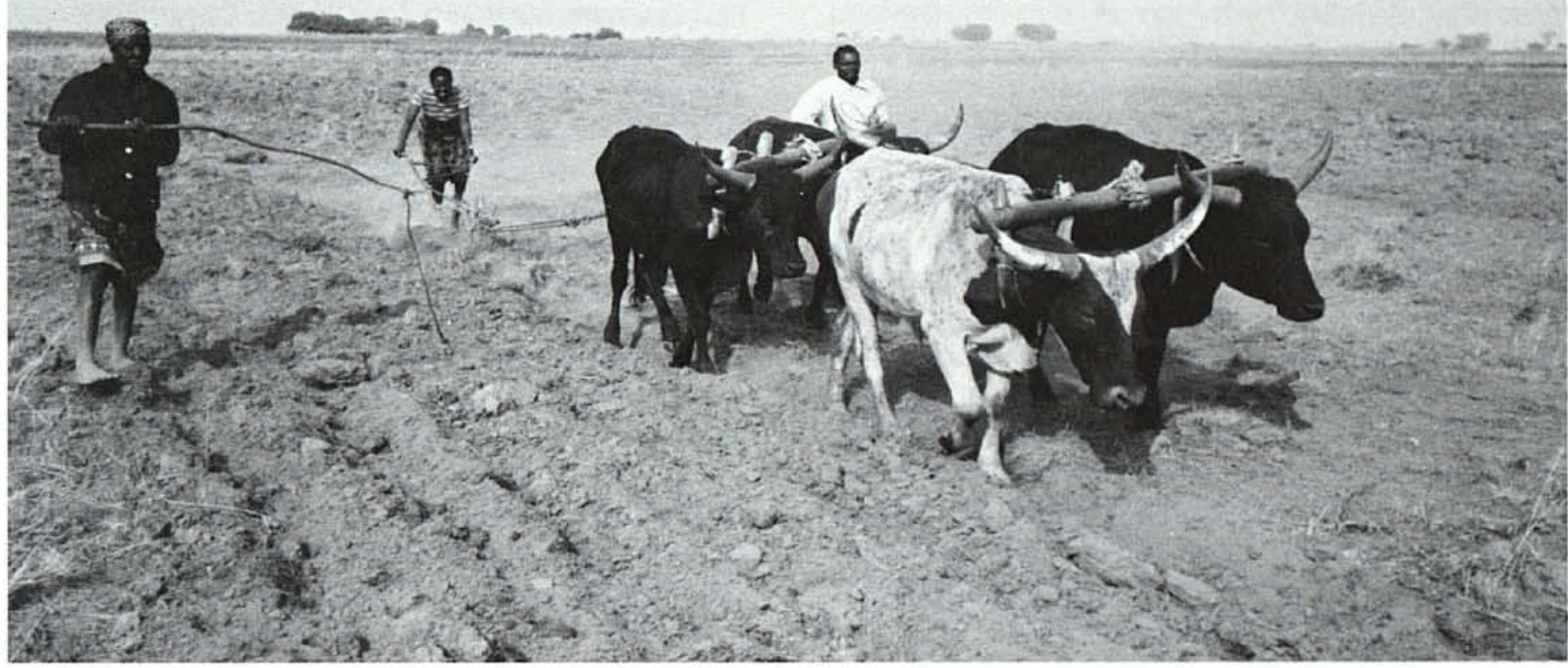

du comité. Le coordinateur du WEP ajoute: "A présent, ce sont eux qui se tournent vers nous lorsqu'ils ont besoin de notre aide ou s'ils ont une idée. Ils se rendent bien compte que nous aussi avons quelque chose à leur apporter".

Formation itinérante. Le WEP encourage également les collègues du Département à planifier la formation sur le terrain de façon qu'elle soit compatible avec les contraintes en temps, les responsabilités domestiques et le niveau d'éducation des femmes. L'introduction de programmes de formation itinérante offre donc une alternative intéressante aux cours plus traditionnels dispensés dans les centres de formation. Cette formule connait un grand succès tant auprès des formateurs que des femmes. La formation itinérante est assurée par les assistants agricoles qui bénéficient de l'assistance de spécialistes autochtones. Chaque session, qui dure de deux à trois jours, se tient au moment et à l'endroit qui conviennent aux participants et porte sur un sujet spécifique comme, par exemple, l'usage de l'engrais, ou la culture en couloir de maïs hybride. Contrairement aux programmes de formation dispensée dans les centres de formation où l'instruction est purement théorique, la formation itinérante utilise un amalgame de méthodes dont les travaux pratiques dirigés, les questions-réponses, les discussions et les essais sur place de ce qui vient d'être appris.
Au départ, le WEP a insisté pour que soit organisé des cours uniquement pour les femmes en raison de leur réticence à assister à des cours qui comprendraient aussi des hommes. Mais l'objectif a toujours été de faire en sorte que les femmes soient plus intégrées. Comme le dit Mary Masona: "Si les femmes restent entre elles, elles seront toujours mises à l'écart". Le WEP reconnait toutefois qu'il est nécessaire d'organiser des cours uniquement pour les femmes lorsqu'on aborde des sujets qui les concernent tout spécialement comme, par exemple, la préservation et l'entreprosage des aliments, et pour leur enseigner des techniques que les hommes maitrisent déjà et que les femmes aimeraient apprendre, comme l'usage de la traction animale.

Contrôle. Le WEP encourage les assistants agricoles à suivre de près le nombre de femmes qui participent aux cours dispensés dans les centres de formation, aux cours de formation itinérante, aux sorties éducatives et aux démonstrations. L'objectif à atteindre en ce qui concerne la participation des femmes aux cours mixtes, aux sorties éducatives (l'occasion pour les agriculteurs et les assistants de passer en revue ce qui vient d'être appris en s'entrainant sur le champ d'un des participants) et aux démonstrations, est de 50 pourcent et les résultats sont relativement encourageants. Durant la saison 1989-1990, la participation des femmes dans tous 
les cours a atteint près de 40 pourcent. Comme l'explique un agent agricole en poste à Mongu, "A présent, les femmes viennent volontairement aux cours et on n'a plus de soupçons à mon égard même si je rencontre une femme seule dans son champs. Et les femmes m'acceptent car elles savent que je possèdent des connaissances qui leurs sont utiles".

\section{Surmonter des Barrières Tradition- relles : "Labourer? Nous Faisons un Travail qui Etait Tabou"}

Le WEP a connu son premier grand succès en permettant aux femmes d'apprendre à labourer avec des boeufs. Dans le passé, les femmes ne labouraient pas; c'était un travail d'homme et on pensait que le contact d'une femme était porteur de malchance et de maladie. De nos jours, étant donné que de plus en plus de foyers sont dirigés par des femmes, on reconnait volontiers que les femmes doivent également savoir maitriser cette technique.

L'usage de boeufs pour labourer les champs est crucial pour augmenter le rendement des productions mais un certain nombre de barrières culturelles, pratiques et financières empêchent les femmes de recourir à des animaux de trait. Bien que les femmes ne gardent pas le bétail, près d'un tiers de toutes les femmes de la province entre en possession de bétail à un moment donné de leur vie que ce soit par héritage ou comme paiement du prix de la mariée. Néanmoins, la croyance persiste parmi les hommes que l'entretien du bétail n'est pas un travail de femme et que de toute façon elles ne sont pas intéressées à les garder.

L'une des raisons qui empêchent les femmes de posséder du bétail est le coût nécessaire à l'entretien des animaux (embauche de gardiens de troupeaux et de personnel pour changer les parcs d'emplacement, achat de médicaments, etc.). Les femmes qui ont les moyens de faire labourer leurs champs se voient généralement contraintes d'accepter des arrangements complexes concernant l'emprunt d'équipement ou l'embauche de personnel. En conséquence, les champs appartenant aux femmes sont généralement labourés après ceux des hommes-c'est-à-dire moins bien et plus tard -et donnent donc des rendements bien plus faibles.

Pour aider les femmes à apprendre à labourer, le WEP a dû réaliser un travail de coordi- nation très important pour faire avancer les choses entre les différentes divisions concernées, notamment les agents de formation en traction animale, les assistants agricoles et les groupes de femmes rurales (description ci-dessous)

Le premier pas. Au début, l'idée même semblait absurde. Un centre de formation en traction animale avait été établi dans la province en 1983, mais personne n'avait songé à former des femmes. Le personnel non autochtone travaillant au centre avait peur de transgresser des tabous culturels et les assistants agricoles pensaient simplement que les femmes étaient incapables d'apprendre à maitriser une telle technique. Mais avec le lancement du projet de culture du riz dans la région, la charge de travail des femmes était telle qu'une solution allait devoir être trouvée.

Une étude sur les femmes impliquées dans la production de riz menée par la spécialiste hollandaise révéla qu'un certain nombre de femmes possédaient déjà un voire deux boeufs ou qu'elles avaient la possibilité d'emprunter un animal auprès des membres de leur famille. Malheureusement, elles n'avaient aucun contrôle sur l'usage des boeufs et ne savaient pas comment labourer elles-mêmes. Discrètement, quelques agricultrices se sont réunies avec Mme Masona et ses collègues et ont commencé à s'entrainer à labourer à l'aide de boeufs. C'est au cours du concours agricole qui se tint peu de temps après que les femmes firent une démonstration de ce qu'elles avaient appris à la grande surprise des spectateurs. Le premier pas en direction de la formation des femmes en traction animale avait donc été franchi.

Reconnaissance grandissante. Le Département réalisa également que des instruments spéciaux pouvant être tirés par des boeufs, notamment, des cultivateurs et des butteuses, allaient être nécessaires ou les mauvaises herbes empêcheraient la production de riz de s'étendre. En Octobre 1989, on lança un programme pour tester et introduire des instruments pouvant être tirées par des boeufs peu coûteux. Grâce à l'action du WEP, les groupes de femmes sont de plus en plus impliquées à ce programme. A l'heure actuelle, la formation en traction animale fait partie intégrante des cours de formation itinérante qui sont dispensés aux groupes de femmes par les assistants agricoles. De plus en plus de femmes se font embaucher en temps qu'équipe pour labourer les champs des autres. Ces équipes de femmes laboureur sont de plus en plus réputées pour leur travail consciencieux et leur agileté à manier les boeufs. 
Changer les attitudes et les aspirations. Les femmes elles-mêmes sont enthousiastes; elles sont fières d'avoir su acquérir cette technique masculine "difficile". Pour elles, ce qui a vraiment changé c'est la manière dont on les traitent au sein de la communauté. Bien que de nombreuses femmes agriculteurs n'ont toujours pas la possibilité de recourir à des boeufs ou à l'équipement, et ne sont pas en mesure d'obtenir les prêts considérables nécessaires à leur achat, elles participent tout de même aux cours parce que "un jour, qui sait, j'aurais peut-être un boeuf" ou "peut-être ma fille fera mieux que moi, alors je pourrais l'aider à labourer" ou "si le maïs est bon cette année, mon groupe décidera après tout d'acheter une équipe et l'équipement nécessaire".

La possibilité pour les femmes de recourir à la traction animale et les autres changements ne sont pas survenus uniquement à cause de la formation et de la persuasion. En fait, deux innovations opérationnelles à l'intérieur du Département même y ont également contribué : le Projet de participation des populations (PPP) et l'Equipe de planification en recherche adaptive (ARPT).

\section{Aider les Femmes a se Valoriser et a s'Organiser}

Une des leçons apprises par le projet de culture de riz est que la mobilisation et le soutien des groupes est indispensable pour a) fournir un point de contact acceptable d'un point de vue culturel entre les femmes et les assistants agricoles de sexe masculin; b) encourager les femmes à utiliser les services existants; c) développer les capacités des femmes à s'organiser et à faire valoir leurs droits au niveau du village.

Initialement, les activités des groupes de femmes était coordinées directement par le WEP. Plus tard, ces activités furent formalisées et intégrées au Projet de participation des populations (PPP) établi grâce à une subvention de l'Organisation des Nations-Unies pour l'Alimentation et l'Agriculture (FAO) et du gouvernement néerlandais. Toutefois, la coordination du PPP s'avéra difficile pour le WEP étant donné qu'il n'avait ni le personnel, ni le mandat pour prendre une part active aux activités de terrain.

C'est la raison pour laquelle, le Département accepta la proposition de Mme Lydia Ndulu qui travaillait au bureau provincial du Département de devenir la coordinatrice provinciale du PPP. Elle travaille également au sein de la division "Economie ménagère". Avec sa formation en nutrition, en économie ménagère et en horticulture et sa longue expérience dans le département, Mme Ndulu avait suffisamment de poids auprès de ses collègues de sexe masculin pour être acceptée comme un professionnel de l'agriculture et non pas simplement comme le responsable d'un "projet spécial pour les femmes".

Recours aux groupes. Lydia Ndulu est convaincue que la meilleure solution pour les femmes est d'accéder aux services et aux structures de soutien existants en étant organisées en groupes. Mais avant que cela ne puisse se produire, il faut que les femmes prennent conscience de leurs droits, développent leurs capacités à s'organiser et à faire valoir leurs droits et surmontent leur timidité et le sentiment qu'elles sont ignorantes.

Le PPP démarra ses activités en février 1984. Dès 1988, 24 groupes s'étaient constitués dans les trois districts de Kaoma, Mongu et Kalabo. Et, vers mi-1990, il y avait 130 groupes comprenant au total 1144 femmes et 507 hommes. Bien que certains groupes étaient composés uniquement de femmes, la plupart d'entre eux comprenaient également des hommes. Au départ, les hommes dans les villages étaient très contrariés par l'importance particulière accordée aux femmes: "Pourquoi est-ce que vous nous tenez à l'écart?". Comme le souligne Mme Ndulu, "C'était vraiment dur à expliquer". Mais le PPP a trouvé qu'à long-terme il était plus avantageux pour les groupes d'incorporer également des hommes si ce n'est que pour donner aux assistants agricoles la garantie qu'ils ne transgressent pas de tabous culturels lorsqu'ils travaillent avec les groupes. De plus, les membres hommes peuvent également défendre les intérêts du groupe auprès d'autres hommes et étant donné qu'ils ont plus facilement accès aux responsables hommes, ils sont mieux placés pour promouvoir les causes des femmes. Toutefois, le PPP a pris des précautions pour éviter que les hommes n'accaparent le pouvoir: aucun homme ne peut élu à la tête d'un groupe.

Les femmes parlent aux femmes. Des femmes de la région ont été recrutées pour travailler comme animatrice de groupes dans les villages. Leur rôle est d'appuyer les groupes de femmes agriculteurs et de rallier d'autres femmes. Une animatrice de groupe de Limulunga, qui est avec le PPP depuis le tout début, se souvient qu'au départ c'était très difficile : "Lorsque nous nous rendions à un village pour parler au chef du village et au chef de section du Parti, j'étais intimidée et ne savais pas comment leur expliquer ce que nous avions l'intention de faire. Nous leur 
disions que le projet était pour des "femmes qui sont une peu lente d'esprit" mais ils avaient des doutes. De plus, nous n'avions pas la moindre idée sur la façon de procéder pour mobiliser les femmes et les soutenir." A présent, elle est convaincu que les femmes dans les villages préfèrent toujours s'adresser en premier lieu à une autre femme et si possible à une femme du miême milieu qu'elles. "Elles ont beaucoup de problèmes qu'elles n'osent pas aborder avec les hommes. Elles peuvent me poser des questions sur la manière de nourrir leurs enfants, sur les outils et les techniques agricoles sans avoir le sentiment d'être stupide ou ignorante".

Lydia Ndulu confesse que bien que cela ait été difficile au départ, les hommes du Département ont fini par accepter. Initialement, ils considéraient l'approche de groupe et l'accent mis sur les femmes commes des "idées FAO" mais "ils ont fini par réaliser que c'était dans l'intérêt du département et que cela facilitait la tâche de tous les gens impliqués".

\section{Impact : “Nous Ne Voulons Pas Mourir de Faim"}

Rosemary Ntoka est chef d'un groupe à Lilu, un village dans la région de Limulanga situé dans le district de Mongu. Rosemary, qui est agricultrice, a deux enfants et attend son troisième. Est-elle heureuse avec le PPP? "Oui, bien sûr! Pour survivre, nous, les femmes, devons acquérir de nouvelles idées et connaissances. Nous ne voulons pas mourir de faim! Et je m'intéresse au groupe parce que de cette manière, nous partageons nos problèmes, nous partageons nos idées et nous apprenons ensemble."

Quelques succès. Dans le village de Kweseka, l'un des groupes les plus anciens connait un grand succès. Le groupe a démarré en 1984 avec neuf membres femmes. Les premières économies que les femmes ont réalisé en temps que groupe provenaient d'un champs de maïs qu'elles cultivaient ensemble. Après le maïs, elles ont essayé le blé, puis à nouveau le maïs, les patates douces et les noix de cajou. Pour la première fois de leur vie, elles ont reçu de l'aide de la part de l'assistant agricole et ont appris à labourer avec des boeufs.

En 1988, le groupe de Kweseka a ouvert un petit commerce avec un investissement de 200 Kw. (20 dollars US) provenant du compte épargne du groupe. En 1990, cette activité leur a permis de générer un profit de $4000 \mathrm{Kw}$. (105 dollars
US). Le groupe fabrique et vend également des balais, des paniers et des bérets en laine. Les économies du groupe sont déposées sur un compte épargne postal réservé pour les investissements et fonds de roulement futurs. (On encourage également les membres du groupe à ouvrir chacun un compte à leur nom). Le groupe a déjà un nouveau projet en tête : se lancer dans l'achat et la vente de maïs raffiné dès qu'elles auront économisé suffisamment d'argent.

Quelques difficultés. Ishekendo, un autre groupe de la région a du mal à s'en sortir. Le village manque d'eau et les possibilités au niveau de l'agriculture ne sont pas très nombreuses. Les femmes ont essayé de cultiver du riz mais la culture a été infesté de charançons. Elles aussi ont appris comment labourer mais elles n'ont pas la possibilité d'emprunter des boeufs et l'équipement nécessaire et elles ont peur d'acheter leurs propres animaux car elles redoutent les voleurs qui rôdent la nuit sur la route toute proche. Avec l'aide de leur animatrice de groupe, elles ont amélioré la commercialisation de leurs produits artisanaux. Le revenu supplémentaire leur a permis d'acheter les intrants nécessaires à la culture du maïs et, lorsque les pluies sont bonnes, elles obtiennent de bonnes récoltes. Bien que jusqu'à présent leurs gains aient été modestes, les femmes sont positives: "Le fait d'être ensemble nous remonte le moral. L'assistant agricole ici est une dame et elle nous encourage énormément. Elle nous a appris à faire du gari (un plat ouestafricain à base de flocons de manioc séchés). C'est épanouissant d'acquérir de nouvelles connaissances. Personne ne nous parlait jamais avant."

\section{Aborder les Priorites Agricoles des Femmes}

Un nombre croissant d'études ont démontré qu'un manque de connaissances sur la façon dont les petits paysans, femmes ou hommes, gèrent leur exploitation diminue l'efficacité et les effets de la recherche agricole. Pour développer ces connaissances, une équipe de planification en recherche adaptive (ARPT) a été établi dans la Province Occidentale. ARPT fait partie d'un programme national lancé par la division Recherche du Département de l'Agriculture (voir Schéma 1) et a pour mission de : a) travailler avec les petits agriculteurs et tous ceux qui veulent le devenir pour leur fournir l'information technique et économique nécessaire pour améliorer leur production; et b) mener la recherche adaptive en 
travaillant directement avec les paysans sur leurs propres champs.

L'équipe travaille en collaboration étroite avec les assistants de recherche (tous des hommes) basés sur le terrain et avec les assistants agricoles. Dès son commencement en 1985, ARPT a également eu pour mission d'inclure les femmes agriculteurs dans son travail et d'accorder une attention toute particulière aux femmes exploitant seules leur ferme. On partait du principe que les femmes travaillant la terre pouvaient être soumises à des contraintes différentes ou avaient des priorités différentes de celles des hommes. Toutefois, en dépit de ce mandat, le sociologue de l'équipe, Gerben Viestra, note que la notion même de "questions de femmes ou de femmes comme nos collaboratrices n'a véritablement pris racine dans la Province Occidentale que vers 1987."

Inclure les femmes aux enquêtes et aux essais. Grâce à l'encouragement du WEP, l'une des premières étapes a consisté à faire en sorte que les enquêtes d'ARPT recueillent également des données sur les femmes chefs de famille et sur les femmes mariées. Un autre effort similaire mais bien plus difficile à réaliser a été d'encourager les assistants agricoles à identifier davantage de femmes prêtes à participer aux essais de terrain. Les connaissances sur le rôle des femmes dans l'agriculture sont malheureusement toujours limitées. Par exemple, de nombreux assistants agricoles ne savent toujours pas que certaines fermes sont exploitées uniquement par des femmes. Et pour leur part, les femmes rurales sont très réticentes à l'idée de mettre en jeu leurs principales cultures vivrières pour tenter des expériences puisque la survie de leur famille souvent dépend du fait d'obtenir au moins une bonne récolte. Les femmes s'intéressent le plus aux expérimentations sur les cultures qui rapportent le plus d'argent (légumes modernes et traditionnels), mais, à ce jour, ces cultures ne figurent pas au rang des priorités de recherche d'ARPT.

Préjugé professionnel. Charles Kapekele Chileya, le socio-économiste d'ARPT, confesse que lorsqu'il a commencé à travailler avec d'autres scientifiques, "j'étais aveugle. J'acceptais la façon de penser des biologistes. Mais, ce qui m'a ouvert les yeux, c'est quand je suis allé faire ma maitrise au Royaume-Uni où nous avions un groupe international comprenant plusieurs étudiantes et enseignantes très engagées et une approche interdisciplinaire. Ces femmes m'ont convaincu que je devrais porter une attention toute particulière à cet aspect de la vie rurale."

A son retour en Zambie, il était un peu déçu de la réaction d'un de ses collègues lorsqu'il lui exposa sa nouvelle façon de voir. Son ami le plus

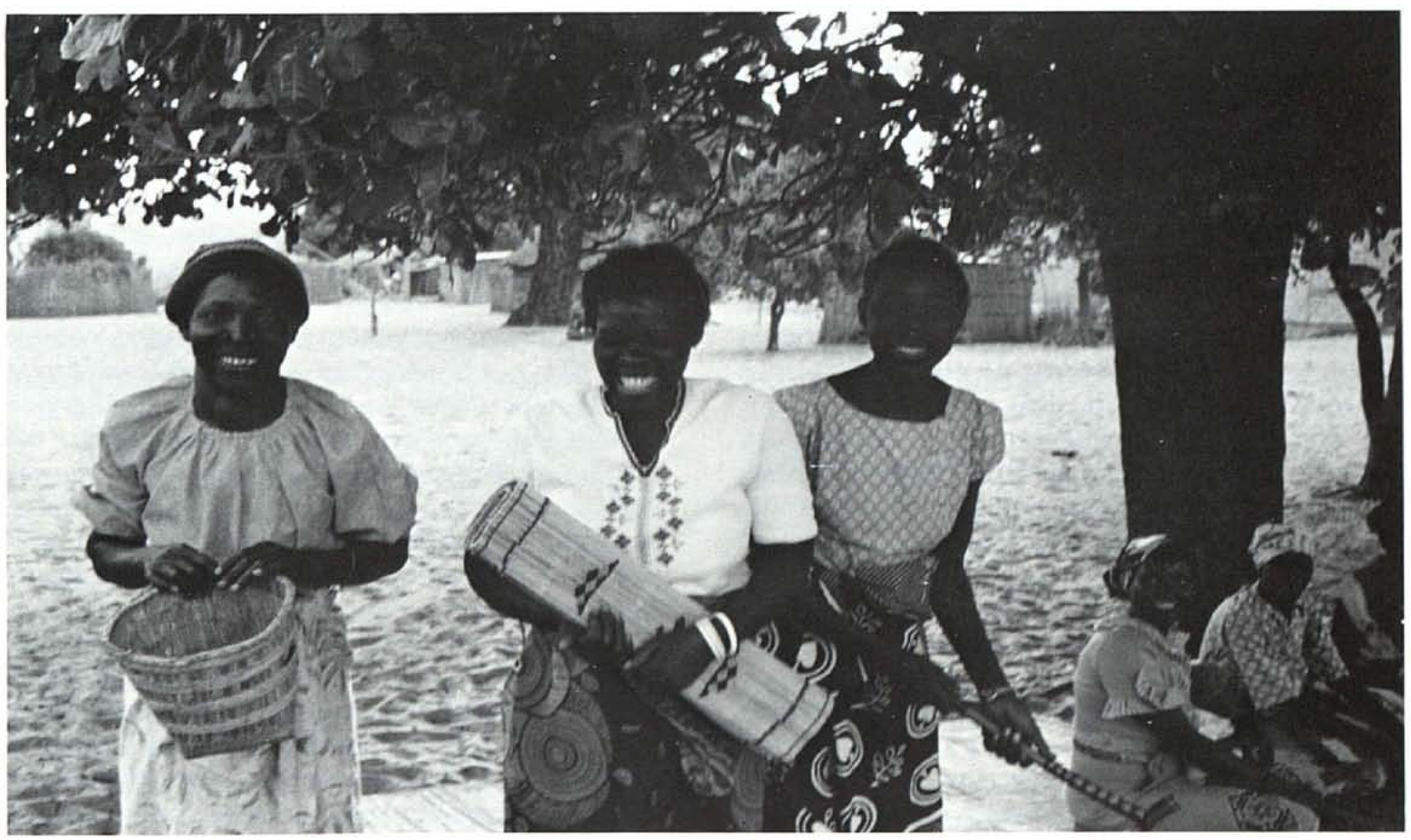


proche, un économiste agricole, lui dit : "Nous ne pouvons pas faire cela. Quelle que soit la catégorie d'agriculteurs, les hommes et les femmes c'est du pareil au même." Après quoi Kapekele Chileya ajouta en se marrant: "Mon ami s'est montré sous son vrai jour lorsqu'il a dit, 'De toute façon, les femmes agriculteurs ne sont pas utiles à l'analyse économique. Tu n'obtiens pas de bons résultats parce qu'elles ne plantent pas à temps!'"

Prendre l'initiative. Vers la fin 1987, le coordinateur provincial d'ARPT, un agronome, avait appris suffisamment pour le convaincre que la participation des femmes était une question à considérer. II demanda à $\mathrm{M}$. Chileya de préparer un rapport à ce sujet qui fut bien reçu par le Département. A présent, on accorde plus d'importance aux bénéfices que les femmes agriculteurs peuvent retirer du travail d'ARPT; par exemple, les cultures typiquement produites par les femmes comme les noix bambara, les haricots, les doliques et l'arachide ont commencé par recevoir de l'attention. "C'est un pas dans la bonne direction et nous devrions continuer comme cela", affirme M. Chileya. "De telles cultures se vendent à des prix élevés et ont une excellente valeur nutritionnelle. Et chaque fois que la femme a besoin d'argent, elle peut en vendre une petite quantité sur le marché ou à ses voisins".

Kapekele Chileya travaille en collaboration étroite avec le WEP pour changer les mentalités au sein du Département. II s'assure, par exemple, qu'au moment de faire la liste des cultures produites par les paysans, l'équipe n'oublie pas de mentionner les cultures "mineures" importantes aux femmes. Et quand il ne peut pas assister à une réunion de personnel, il demande à Mary Masona ou Lydia Ndulu d'y aller à sa place. Quand un assistant agricole invite l'équipe à participer à une sortie éducative, il insiste pour que ARPT n'y aille que dans le cas où des femmes agriculteurs seront présentes également.

M. Chileya encourage aussi ARPT et les assistants de recherche à travailler avec les groupes du PPP. Procéder à des essais expérimentaux avec les groupes de femmes est non seulement avantageux pour ARPT mais également pour les groupes puisque cela leur permet de consolider leur organisation et d'accroitre les gains provenant de la vente de leurs récoltes.

Reconnaitre la diversité. Kapekele Chileya estime toutefois que si ARPT a l'intention de prendre les femmes agriculteurs au sérieux, il faudra arrêter de parler de "petits paysans" en se référant aux hommes ou même de "femmes chefs de famille". "ARPT doit reconnaitre que la majorité de ses clients (potentiels) sont des femmes. Mais elles ne gèrent pas toutes leur exploitation de la même façon ni avec les mêmes ressources. Nous devons être beaucoup plus prudent sur notre manière de définir le qui fait quoi, et pour savoir exactement quels sont leurs besoins et leurs possibilités. Nous devons reconnaitre que les "femmes" ne sont pas toutes pareilles: leurs besoins sont différents".

\section{Le Processus de Changement Bureaucratique}

Un élément clé pour changer les attitudes à l'intérieur du département et développer des activités ciblées spécialement vers les femmes rurales fut l'appui donné par le responsable agricole provincial en poste au moment où le WEP démarrait ses activités. II a défendu et protégé l'initiative et à aider à établir les "questions touchant aux femmes rurales" comme une préoccupation légitime et digne d'intérêt. "Sans lui, nous n'aurions jamais pu ouvrir les portes", dit Mary Masona. Promu à un poste de haut-fonctionnaire au sein du Ministère, l'ancien responsable agricole provincial s'arrange désormais pour que les succès et les difficultés du WEP et les besoins des femmes rurales soient abordés dans le cadre de la politique nationale.

L'importance des données. D'un autre côté, le responsable agricole provincial adjoint confesse qu'il a travaillé dans la vulgarisation agricole pendant de nombreuses années sans jamais considéré qu'il était nécessaire, voire utile, de mettre en place des programmes spéciaux à l'intention des femmes. Toutefois, en 1985, après avoir participé à plusieurs séminaires consacré aux femmes et à l'agriculture, il acquit la certitude que des programmes ciblés étaient indispensables. "Au début, je pensais que les séminaires allaient simplement refléter les opinions de quelques personnes. Mais les données des enquêtes m'ont convaincu. Pour la première fois nous avions quelque chose de concret sur quoi nous fonder. A présent, je lis systématiquement tout ce que publie le WEP ou l'ARPT sur les activités agricoles des femmes dans la province".

En avril 1990, le responsable agricole provincial adjoint a mené une petite enquête à échantillon aléatoire dans deux régions du district de Mongu pour évaluer le travail des assistants agricoles. II a trouvé qu'aucun des "agriculteurs de liaison" choisis par les assistants agricoles 


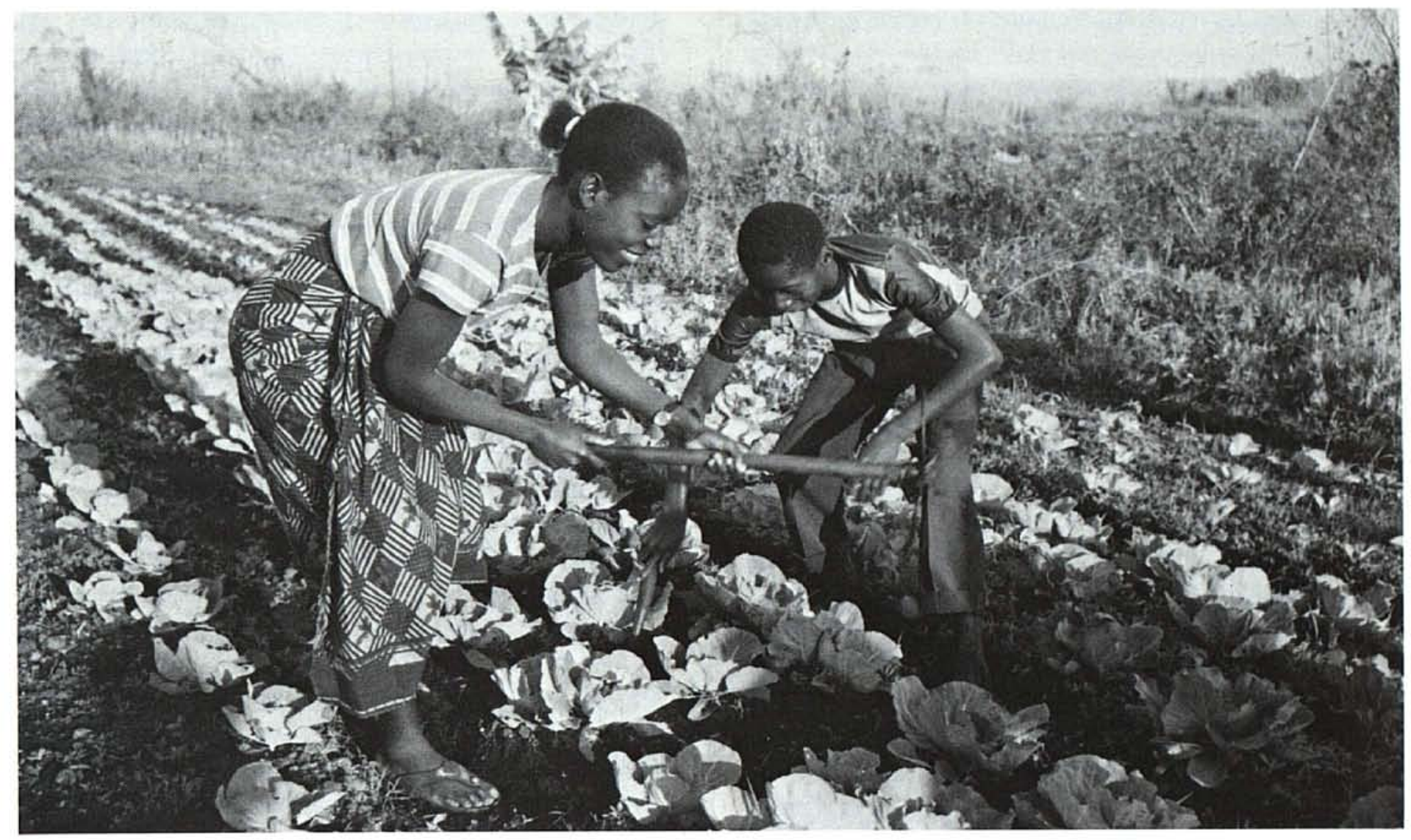

comme leur principal point de contact avec les communautés paysannes n'étaient des femmes. "Cette situation est imputable aux critères de sélection des paysans", remarque-t'il. "La plupart des femmes rurales n'ont pas les indicateurs nécessaires pour être choisies. Peut-être devrionsnous revoir les indicateurs". Mais, ajoute-t'il, "Personnellement, je pense que l'approche de groupe est la solution". Non seulement les groupes permettent de rendre le travail des assistants agricoles "moins cher par heure de contact" mais, selon lui, étant donné la fragilité des ressources du département, les femmes auront une meilleure chance de conserver leurs gains durement acquis si elles possèdent des structures à travers lesquelles elles peuvent devenir quelque peu indépendantes.

L'approche de groupe. Les effets de l'approche de groupe sur la vulgarisation agricole et la formation ont impressionné systématiquement tous les collègues du WEP travaillant dans le Département. Le responsable agricole du district de Mongu affirme: "Nous avons eu ample opportunité de nous rendre compte que la notion "d'animatrice de groupe" est très efficace. A présent, nous voyons tous les jours des femmes, et des hommes, former des groupes eux-mêmes et ils sont encouragés dans cette initiative par les assistants agricoles. Dans le passé, c'était les femmes qui faisaient le travail et les hommes qui recevaient la formation".

Voir c'est croire. Le responsable agricole du district a lui-même changé d'opinion vers 1982-83 lorsqu'il travaillait comme conseiller en élevage et vit les femmes labourer pour la première fois. Aujourd'hui il est convaincu : "Si elles ont accès à des boeufs et à l'équipement, en particulier les femmes qui n'ont pas de mari, elles peuvent planter à temps et améliorer le rendement de leurs productions... A présent, lorsqu'il y a des concours agricoles, les autres sont impressionnés quand ces femmes viennent avec leur groupe montrer ce qu'elles ont cultivés. Elles gagnent beaucoup de prix! Cela a un effet positif sur la façon de penser des indunas (les chefs traditionnels) et des gens."

\section{Creer des Reseaux}

Le WEP sait qu'il a besoin de l'appui d'un groupe plus large de collègues. Très souvent, les activités touchant aux femmes sont marginalisées même au sein d'une administration favorable à la poursuite de telles activités. C'est pourquoi, il est nécessaire d'établir des relations personnelles avec des hauts-fonctionnaires et de mettre en place des réseaux de communication pour favoriser la discussion et l'échange d'idées. Pour légitimiser les questions touchant aux femmes et à 
l'agriculture au sein de l'administration, il est particulièrement important de prendre les deux initiatives suivantes : 1) établir des comités de développement des femmes qui rassemblent les femmes travaillant dans divers secteurs de l'administration et qui sont soucieuses de faire avancer les choses; 2) mettre en place des ateliers parrainés par le gouvernement à l'intention des cadres et du personnel technique travaillant au niveau de la province et des districts en vue d'échanger des informations et d'évaluer leurs expériences sur les activités agricoles des femmes.

\section{Evaluer les Couts}

Le budget du Bureau du Département de l'Agriculture au niveau de la province est très limité. Le gouvernement dépense en moyenne entre 400000 et 500000 kwacha (entre 100000 et 130000 dollars US) chaque trimestre pour couvrir les frais généraux des activités de vulgarisation agricole du département. Les projets financés par des sources extérieures contribuent à peu près cinq fois autant au budget total du Département. Néanmoins, le succès de tout projet dépend de la capacité de prise en charge des ressources de base par le département lui-même.

Le budget total du PPP de 1986 à la fin de l'année 90 s'élevait à 248000 dollars US. Le département prend en charge les salaires des coordinateurs au niveau de la province et des districts, une partie des frais d'essence et de formation, les indemnités de subsistance journalières des assistants agricoles et d'un chauffeur.

Les frais annuels du PPP par district sont modestes. Par exemple, les dépenses dans le district de Mongu de janvier à décembre 1989 se montaient à 257169 kwacha (moins de 7.000 dollars US). Bien que le projet ait remis à chacun des coordinateurs de district un vélomoteur, ce moyen de locomotion est inutilisable particulièrement au moment de la saison des pluies. Les agents du PPP sont donc souvent obligés de se faire emmener en voiture par le personnel du responsable agricole du district mais lorsque les véhicules sont en panne, les agents du PPP ne peuvent pas aller travailler non plus.

Le Département prend en charge le salaire du coordinateur et les frais de bureau du WEP. Entre 1983 et 1988, les frais du WEP furent absorbés par le programme de vulgarisation agricole et s'élevaient approximativement à 120.000 dollars US par an. De 1989 à la fin 1991, le budget total se situait entre 550000 et 600000 dollars US. Dans ce montant sont également inclus les salaires des chercheurs et des consultants internationaux.

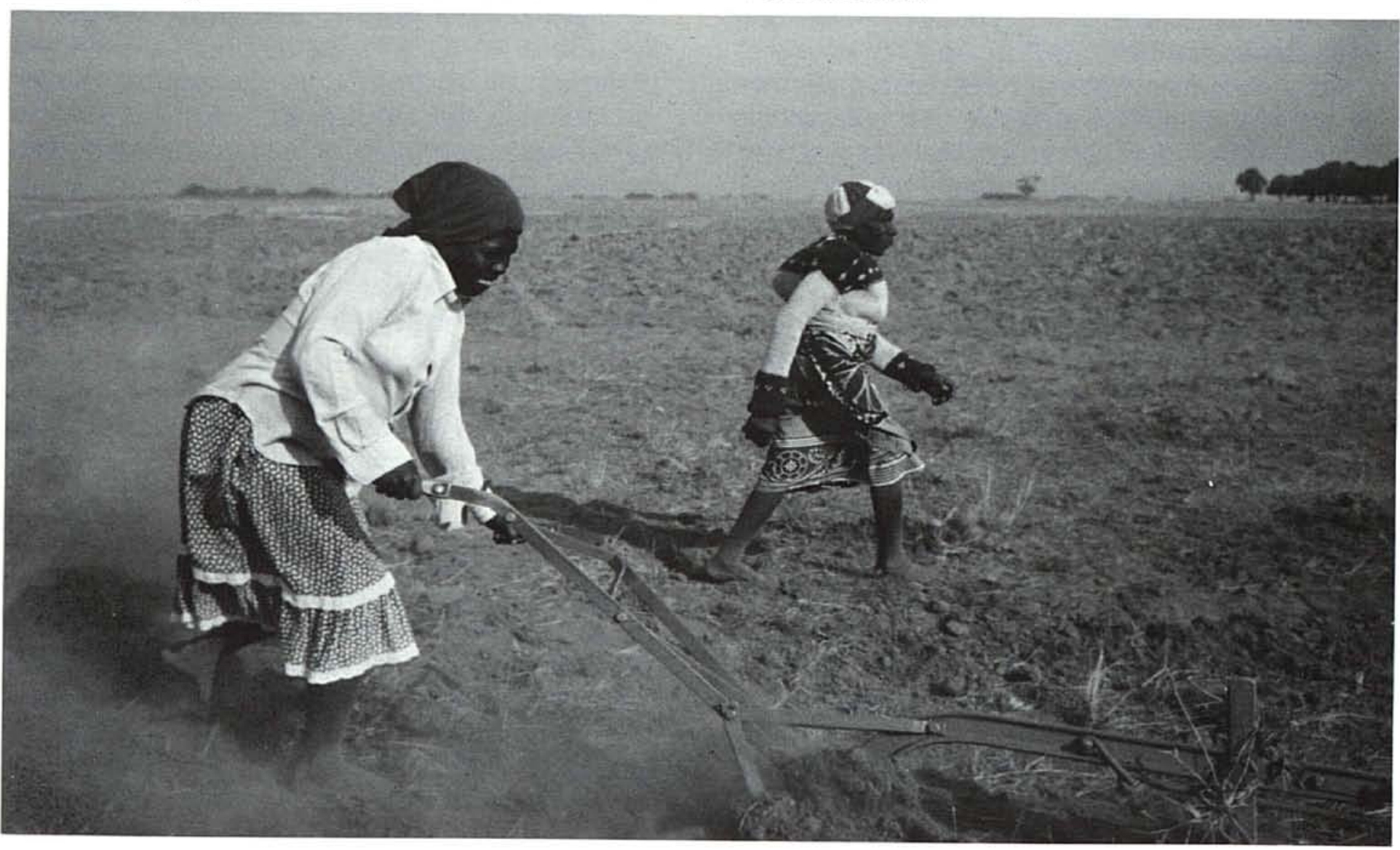


Le PPP et le WEP ont tous deux donné au personnel de sexe masculin et féminin du département l'occasion de mieux se familiariser avec la situation des femmes agriculteurs dans leur région et à l'échelon international. Le responsable agricole provincial pense que cette conscientisation du personnel est ce qui a aidé le département à modifier sa ligne de conduite puisqu'il réalise désormais que le fait de négliger les femmes entrave le développement de l'agriculture -un problème partagé par de nombreux payset que des solutions pratiques sont possibles.

\section{Directions Futures}

Dernier en ligne quand les ressources sont rares. Les problèmes majeurs auxquels s'est heurté le programme n'ont pas été, comme on le pensait initialement, des problèmes dûs à la mentalité des différents participants mais plutôt des problèmes d'ordre logistique. En effet, les obstacles liés aux coutumes, à l'attitude, aux rapports hommes-femmes, etc. se sont avérés en fait beaucoup plus faciles à surmonter que le manque de ressources financières qui inhibent le développement de l'ensemble des activités du département. Quand les ressources sont rares, le WEP et les activités dont il a la charge viennent en fin de liste des priorités. Par exemple, il n'y a pas suffisamment de véhicules, l'essence est chère voire quelquefois introuvable et l'entretien est difficile à assurer. L'appui aux groupes et aux assistants agricoles est de ce fait moins fréquent ou moins fiable qu'on aimerait. Ces problèmes de transport ont pour effet d'entraver l'expansion des activités vers de nouvelles régions et groupes d'agriculteurs et ont limité le nombre de fois que les assistants agricoles et autres membres du personnel ont pu obtenir des cours de formation sur les activités agricoles des femmes.

Problèmes de personnel. La poursuite du développement de nouveaux groupes est rendue difficile en raison d'un problème de personnel. En effet, les animatrices de groupes dont le rôle est de mobiliser et d'organiser les femmes agriculteurs en groupes ne peuvent pas être absorbées dans la structure gouvernementale parce que leur niveau d'éducation est trop bas et pourtant elles sont indispensables au bon fonctionnement du système. Le responsable agricole Provincial Adjoint soulève également un autre problème de personnel dont il a souvent discuté avec ses collègues de sexe féminin travaillant au siège : trop peu de femmes posent leur candidature pour des postes auprès du département en partie parce que le nombre de femmes diplomées en agriculture est très faible. "C'est là où intervient le WEP", fait remarquer Mary Masona. "Nous devons simplement convaincre nos collègues de sexe masculin de travailler avec les femmes agriculteurs et leur donner les moyens de pouvoir le faire."

\section{Accomplissements}

La principale réussite du WEP a été d'enclencher un processus de changement interne au sein d'une administration agricole très conservatrice. Pour atteindre ce résultat, le WEP s'est principalement attelé à modifier le comportement et la mentalité des collègues de sexe masculin travaillant dans les divisions les plus importantes du Département de l'Agriculture (Elevage et Equipements, Formation, Vulgarisation Agricole et Recherche). L'expérience démontre qu'avec un personnel dévoué et une vision claire des objectifs stratégiques, le concensus est possible même lorsque l'initiative vient d'une division sans grand pouvoir et relativement marginalisée comme la division "Economie ménagère".

\section{Leçons à Retenir}

1. Pour aboutir à une intégration efficace des femmes agriculteurs dans les programmes de vulgarisation agricole dispensés par une administration généralement très conservatrice (dominée par les hommes), il est nécessaire d'adopter un éventail d'approches différentes. La persuasion, la concertation, l'échange d'information et les démonstrations preuve à l'appui sont tous des outils indispensables pour redéfinir les mandats, mettre sur pied des programmes de formation de façon à susciter une meilleure compréhension des activités et des contraintes propres aux femmes en agriculture, élargir la portée thématique des programmes de recherche, mettre en place des services techniques ciblés vers les femmes et encourager et soutenir les femmes agriculteurs à accroitre leurs possibilités et leurs connaissances.

2. Lorsqu'il y a suffisamment de soutien de la part de ceux qui occupent les échelons les plus élevés dans l'administration, il est possible même à une petite équipe opérant à partir d'une division relativement marginalisée d'accomplir des changements. Le leadership du responsable agricole de la province occidentale ainsi que le dévouement et la détermination des principaux responsables de sexe féminin de la division "Economie ménagère", du WEP et du PPP ont crée un climat propice à l'établissement et au 
maintien de relations de travail positives avec d'autres divisions chargées d'assurer les activités de vulgarisation agricole. L'expérience du WEP démontre qu'il n'est pas nécessaire de disposer de sommes considérables ou encore de recruter toute une armada de personnes pour opérer des changements significatifs à partir du moment où la volonté politique existe.

3. Bien que le processus de changement interne est long et souvent laborieux, il est essentiel d'encourager une telle évolution au sein d'une large administration et dans le même temps créer le besoin pour des changements à l'extérieur. La nécessité d'intégrer les femmes aux programmes de vulgarisation est un problème que le Départment de l'Agriculture a lui-même identifié. De ce fait, le département a pu s'enorgueillir de son rôle de chef de file en tentant de mieux répondre aux besoins des femmes agriculteurs. Le recours à une approche qui favorise une attitude ouverte au changement plutôt qu'une attitude défensive est sans doute un moyen plus approprié pour accomplir des changements, plus particulièrement lorsque les ressources sont très limitées. Dans le même temps, créer une demande à la base est cruciale pour garantir la mise en place de programmes appropriés et efficaces une fois que l'administration sanctionne l'implantation de tels programmes.

4. Outre la formation et la concertation, il est indispensable de disposer de données statistiques et de preuves concrètes pour convaincre les responsables des politiques en la matière et les planificateurs de prendre l'agriculture féminine au sérieux - voir c'est croire! Ceci souligne l'importance d'associer les femmes aux travaux de recherche, aux essais sur le terrain et aux collectes de données pour obtenir des informations précises sur ce que les femmes agriculteurs font, ce qu'elles sont capables de faire et en quoi leurs besoins diffèrent de ceux des hommes.

5. Rassembler les femmes en groupes est un moyen efficace pour les aider à accéder aux activités de vulgarisation agricole, les encourager à prendre de nouvelles initiatives et obtenir l'accès aux ressources et conférer un cadre au sein duquel elles peuvent bénéficier de soutien et d'encouragement mutuels-ce qui particulièrement important étant donné que de plus en plus de femmes sont le seul soutien économique de leur famille et que les liens familiaux et communautaires traditionnels sont de plus en plus fragilisés. Le processus de groupe crée également un environnement sécurisant qui aident les femmes à mieux définir et articuler le type d'assistance dont elles ont besoin même lorsque leurs opinions diffèrent de celles de l'agent de vulgarisation de sexe masculin!

6. Etant donné que la plupart des agents de vulgarisation agricole sont des hommes et que les ressources qui permettraient de former et d'embaucher des agents de sexe féminin sont quasi-inexistantes, les agents de vulgarisation de sexe masculin peuvent procurer des services efficaces aux femmes agriculteurs s'ils bénéficient d'une formation qui les sensibilisent sur le rôle et les besoins des femmes, s'ils sont encouragés dans leurs initiatives par des collègues qu'ils respectent (particulièrement d'autres hommes) et s'ils peuvent constater par eux-mêmes ce que les femmes sont en fait capables de faire. Par ailleurs, l'établissement de groupes de femmes agriculteurs facilitera la tâche des agents de terrain de sexe masculin qui seront moins réticents à travailler avec des groupes qu'en tête-à-tête avec des femmes qui ne sont pas des membres de leur famille.

7. Bien que les femmes sont avant tout des agriculteurs et que la préocupation principale est de garantir leur accessibilité aux activités de vulgarisation agricole, le développement d'activités génératrices de revenus non agricoles comme, par exemple, la fabrication et la commercialisation de produits artisanaux peut en fait être une stratégie acceptable d'un point de vue culturel pour organiser les femmes en groupes et leur procurer une source de revenu dont elles pourront se servir pour investir dans leur exploitation agricole. Bien que la pratique d'une telle activité est innoffensive et peut présenter des avantages, il faut veiller à ce qu'elle n'éclipse pas les activités agricoles des femmes.

8. Les femmes sont tout à fait capable d'acquérir des techniques non traditionnellescomme par exemple le recours à la traction animale-à partir du moment où elles bénéficient du soutien et de la formation nécessaires. Une fois qu'un petit groupe de femmes a prouvé qu'elles savent maitriser une activité que seuls les hommes exerçaient et qu'il apparait que l'accès à cette activité leur permettra de générer une augmentation considérable de leur productivité (et donc du bien-être de la famille toute entière) sans pour autant compromettre le rôle des hommes, les anciens préjugés et tabous sur ce que les femmes peuvent et doivent faire vont peu à peu disparaitre.

9. Une dépendance minime sur des fonds et du personnel venant de l'étranger peut certes faciliter la prise de contrôle interne du processus 
de changement au sein d'une administration. Toutefois, la validation externe d'un concept innovateur comme, par exemple, reconnaitre et soutenir le rôle des femmes en agriculture peut être un facteur crucial qui confère la légitimité aux efforts entrepris au niveau local dès le tout début. Dans la province occidentale, ceci fut démontré par la présence initiale d'un expert international de sexe féminin et par l'exposition de M. Chileya à de nouveaux concepts durant son séjour au Roy-
aume-Uni et son retour en Zambie comme un partisan en faveur du changement. La prise de conscience de l'existence de nombreux ouvrages et d'expériences sur les activités agricoles des femmes peut être particulièrement utile aux programmes désireux d'identifier et de répondre aux besoins des femmes en agriculture et les aider à tirer les leçons d'expériences menées dans d'autres endroits.

\section{Schéma 1 \\ Organigramme du Département de l'Agriculture}

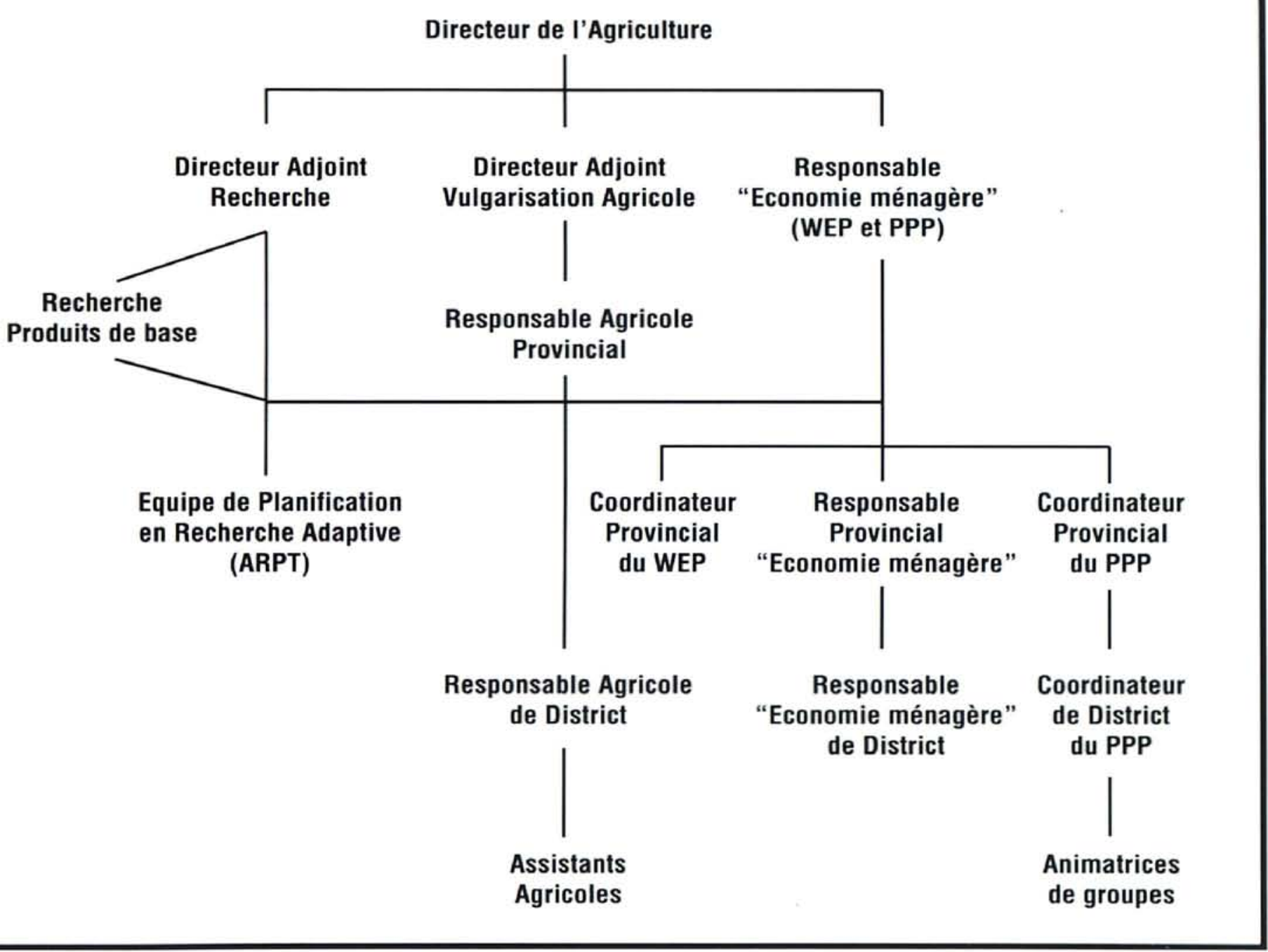




\section{Annexe}

Les publications suivantes donnent des indications sur les approches susceptibles d'aboutir à une intégration efficace des femmes agriculteurs dans les programmes de vulgarisation agricole. Pour obtenir des renseignements sur le coût des publications et les frais de port, veuillez contacter directement la maison d'édition. En fin de liste figurent également les coordonnées de deux programmes qui offrent des cours de formation à court-terme sur la façon de travailler avec les femmes agriculteurs en Afrique.

\section{Publications}

1) Working Together: Gender Analysis in Agriculture, Vol 1, Case Studies; Vol 2, Teaching Notes, par Hilary Sims Feldstein et Susan V. Poats, éditrices; 2) Women's Roles and Gender Differences, série de sept études de cas africaines et de deux études de cas asiatiques à l'intention des planificateurs de programmes; 3) The Impact of Male Outmigration on Women in Farming par Ingrid Palmer. (The Kumarian Press, 630 Oakwood Avenue, Suite 119, West Hartford, CT 06110-1529, EtatsUnis d'Amérique. Téléphone : (203) 953-0214 Télécopie : (203) 953-8579)

Women in Agriculture; What Development Can Do, par Myra Buvinic et Rekha Mehra, 1990. (ICRW, 1717 Massachusetts Avenue, N.W., Suite 302, Washington, D.C. 20036, Etats-Unis d'Amérique)

The Gender Variable in Agricultural Research, par H.S. Feldstein, C.B. Flora et S.V. Poats (Women in Development Unit, IDRC, Ottawa, Canada)

Using Male Research and Extension Personnel to Target Women Farmers, par Anita Spring, 1987 (Women in International Development, Michigan State University, Working Paper N. 14)

Agricultural Extension for Women Farmers in Africa, par Katrine A. Saito et C. Jean Weidemann, Documents de synthèse de la Banque Mondiale, No 103F, 1990. (World Bank Publications, Building 424, Raraitan Center, 80 Northfield Avenue, Edison, NJ 08818-7816, Etats-Unis d'Amérique. Téléphone : (908) 225-2165 - Télécopie : (908) 417-0482)
Women and Livestock Production in Asia and the South Pacific Region, par Alexandra Stephens, RAPA Publications No 1990/5. (Regional Office for Asia and the Pacific, FAO, Bangkok, Thaillande)

Gender Issues in Farming Systems Research and Extension, par M. Schmink, S.V. Poats et A. Spring, éditeurs (Boulder, Colorado : Westview Press, 1988)

\section{Programmes de formation}

Pan African Institute for Development (PAID), Kabwe

East and Southern Africa

P.O. Box 80448

Kabwe, Zambie Contact : Isaac Mungo,

Coordinateur de Cours

Cours de formation à court-terme dans les domaines suivants: activités agricoles des femmes, agents de vulgarisation agricole de sexe féminin, planification de programmes, et gestion et formation.

Eastern and Southern Africa Management Institute (ESAMI)

P.O. Box 3030

Arusha, Tanzanie

Contact : Hilda Tadria

ou

P.O. Box 56628 Nairobi, Kenya

Cours de formation à court-terme sur la planification des activités de développement, la gestion et les femmes-une perspective africaine, les femmes et le marketing, les femmes et la méthodologie de recherche, les femmes et la formation. 
Mise en page: Ann Leonard

Typographie: Village Type and Graphics

Photos: Janice Jiggins

Imprimeur: Graphic Impressions, Inc.

Traduction: Pia Heitz

\section{Les Numéros de SEEDS Disponibles en Français}

No. 3 "Les Coopératives des Vendeuses de Marché: Accorder Crédit aux Femmes" par Judith Bruce (Nicaragua)

No. 4 "Les Femmes et L'Artisanat: Mythe et Réalité" par Jasleen Dhamija (International)

No. 5 "La Coopérative de Markala: Une Nouvelle Approche des Rôles Economique Traditionnaux" par Susan Caughman et Mariam N'diaye Thaim (Sénégal)

No. 6 "Le Forum pour les Femmes Actives: Lutte pour le Crédit et le Changement," par Marty Chen (Inde)

No. 7 "Creér des Emplois pour Les Femmes dans des Activités de Production Non-Artisanales au Bangladesh" par Marty Chen (Bangladesh)

No. 10 "La Conservation des Forêts au Népal: Encourager la Participation des Femmes" par Augusta Molnar (Népal)

Nous attendons vos remarques, commentaires et idées de projets à publier dans les prochains numéros de SEEDS. Si vous souhaitez recevoir des exemplaires supplémentaires de ce numéro ou faire partie de nos abonnés, n'hésitez pas à nous écrire. Envoyez votre courrier à:

Ann Leonard, Conseillère à la Rédaction SEEDS P.O. Box 3923

Grand Central Station New York, New York 10163 U.S.A. 


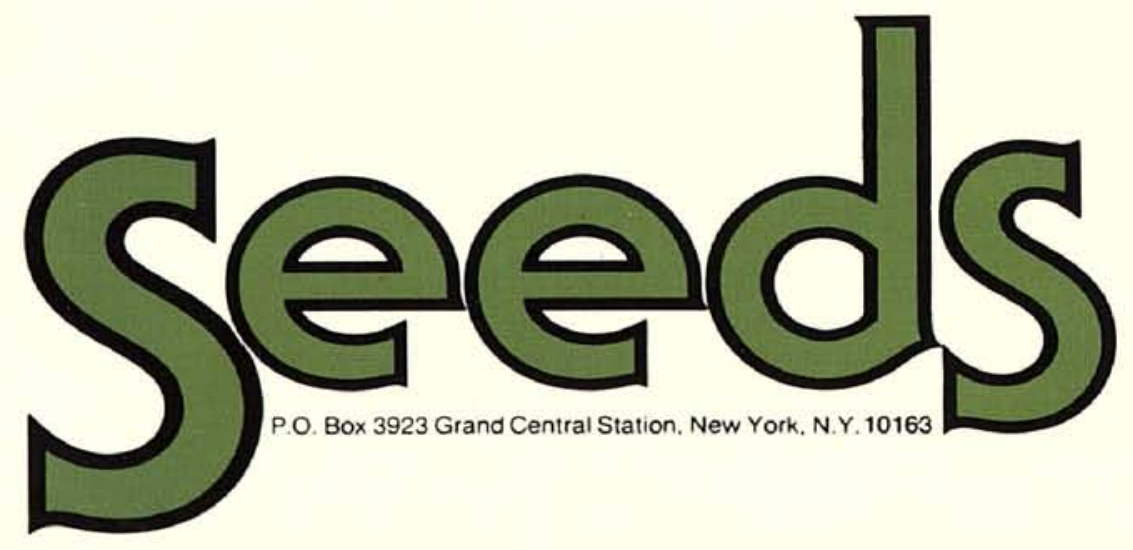

